\title{
Increased migration and motility in XIAP-null cells mediated by the C-RAF protein kinase
}

\section{Lauren G Russell}

Newcastle University

Lydia AK Davis

Newcastle University

Jill E Hunter

Newcastle University Biosciences Institute, Newcastle University

Neil D Perkins

Newcastle University Biosciences Institute, Newcastle University

Niall S Kenneth ( $\nabla$ niall.kenneth@liverpool.ac.uk)

University of Liverpool

\section{Research Article}

Keywords: XIAP, cell migration, C-RAFE protein kinase, cell signalling

Posted Date: September 29th, 2021

DOI: https://doi.org/10.21203/rs.3.rs-923703/v1

License: (c) (i) This work is licensed under a Creative Commons Attribution 4.0 International License. Read Full License 


\section{Increased migration and motility in XIAP-null cells mediated by the C-RAF protein kinase}

${ }^{1}$ Lauren G Russell, ${ }^{1}$ Lydia AK Davis, ${ }^{1}$ Jill E Hunter, ${ }^{1}$ Neil D Perkins and ${ }^{1,2}$ Niall S Kenneth*

${ }^{1}$ Newcastle University Biosciences Institute, Faculty of Medical Sciences, Newcastle University, Newcastle upon Tyne, NE2 4HH, UK. ${ }^{2}$ Department of Molecular Physiology and Cell Signalling. Institute of Systems, Molecular and Integrative Biology, University of Liverpool, L69 7ZB.

*To whom correspondence should be addressed. Email: niall.kenneth@liverpool.ac.uk 
The product encoded by the X-linked inhibitor of apoptosis (XIAP) gene is a multi-functional protein which not only controls caspase-dependent cell death, but also participates in inflammatory signalling, copper homeostasis, response to hypoxia and control of cell migration. Deregulation of XIAP, either by elevated expression or inherited genetic deletion, is associated with several human disease states. Reconciling XIAP-dependent signalling pathways with its role in disease progression is essential to understand how XIAP promotes the progression of human pathologies. In this study we have created a panel of genetically modified XIAP-null cell lines using TALENs and CRISPR/Cas9 to investigate the functional outcome of XIAP deletion. Surprisingly, in our genetically modified cells XIAP deletion had no effect on programmed cell death, but instead the primary phenotype we observed was a profound increase in cell migration rates. Furthermore, we found that XIAP-dependent suppression of cell migration was dependent on XIAPdependent control of C-RAF levels, a protein kinase which controls cell signalling pathways that regulate the cytoskeleton. These results suggest that XIAP is not necessary for control of the apoptotic signalling cascade, however it does have a critical role in controlling cell migration and motility that cannot be compensated for in XIAP-knockout cells. 


\section{Introduction}

The Inhibitors of apoptosis (IAP) proteins are a family of functionally and structurally related proteins predominantly known for the regulation of caspases and immune signalling ${ }^{1}$. In mammalian cells there are eight IAP family members all characterised by the presence of at least one baculovirus repeat (BIR), the domain utilized by viral IAP proteins to compromise host cell death machinery ${ }^{2}$. Of the 8 mammalian IAP family members the X-linked IAP (XIAP) is perhaps the best-characterized, with the most direct links to human disease ${ }^{3,4}$. XIAP contains $3 \mathrm{BIR}$ domains located on the amino terminus of the protein that allow XIAP to inhibit apoptotic cell death. In fact XIAP is the only mammalian IAP protein that has the capacity to directly bind to and functionally inhibit caspases; specifically caspase-3, caspase-7 and caspase-9, to suppress apoptosis ${ }^{5-7}$. In addition to the BIR domains XIAP contains a carboxy-terminal RING domain that provides it with E3 ubiquitin ligase activity, as well as a ubiquitin associated domain (UBA) that can interact with ubiquitin chains (Figure 1A) $8,9,10,11$. This enables XIAP to participate in multiple ubiquitin-dependent signal transduction cascades enabling it to act as a key intermediate in a variety of cellular pathways including, but not limited to: NF-KB signalling 3,12,13, MAPK/JNK signalling ${ }^{14}$, maintenance of intracellular copper levels ${ }^{15-17}$, hypoxia-induced gene expression ${ }^{18}, \mathrm{Wnt} / \beta$ catenin signalling ${ }^{19}$, regulation of autophagy ${ }^{20-22}$ and control of cell motility and migration $23,24-27,28$. Due to its key role in signal transduction pathways, deregulation of XIAP has been implicated in the pathogenesis of human cancers and inflammatory diseases ${ }^{3}$. XIAP is highly overexpressed in many forms of cancer, including breast ${ }^{29}$, renal ${ }^{30,31}$, bladder ${ }^{32}$ and certain haematological malignancies ${ }^{33}$. Indeed, a systematic review correlates high XIAP expression and poor patient outcomes in a variety of solid tumours ${ }^{34}$. Conversely, inactivating genetic mutations in the XIAP gene can result in an extremely rare primary immunodeficiency in humans, Type 2 X-linked lymphoproliferative disease (XLP2), characterised by a defective immune system that is powerfully responsive to infection with the Epstein-Barr virus (EBV) ${ }^{4,35,36}$. It is therefore important to reconcile the molecular properties of XIAP with its role in the progression of human 
disease states to understand the critical roles of XIAP, so as to facilitate the development of novel therapeutics.

To study the cellular function of XIAP we created XIAP-null human cell lines using site directed nucleases and compared their properties with isogenic wildtype controls. Surprisingly, we find no obvious defects in the induction of caspasedependent apoptosis in XIAP-deficient cell lines. Instead, the primary XIAPdependent defect we observe is an increase in cell migration in XIAP-null cells. We find that levels of C-RAF protein kinase are elevated in XIAP-null cells, consistent with increased cell migration rates. Re-expression of XIAP was sufficient to reduce C-RAF levels and suppress the motility of XIAP-null cells. However, introduction of an XIAP mutant that does not have E3 ligase activity did not reverse the invasive phenotype, indicating that control of cell migration by XIAP relies on its ability to ubiquitinate substrates. Together our results suggest that XIAP is critical for modulating cell motility in a manner dependent on its ability to conjugate ubiquitin to target substrates. 


\section{Materials and Methods}

\section{Cells}

U2OS and HeLa cells were sourced from the ATCC and grown in DMEM supplemented with $10 \% \mathrm{FBS}, 1 \% \mathrm{~L}$-gluatamine, and $1 \%$ penicillin streptomycin at $37^{\circ} \mathrm{C}$ and $5 \% \mathrm{CO} 2$. Cells were routinely tested for contamination.

\section{Plasmids}

pEBB-HA, pEBB-HA-XIAP, pEBB-HA-XIAP-D148A/W310, pEBB-HA-XIAP-F495A were kind gifts from Colin Duckett (Duke University). CRISPR/ Cas9 and TALEN constructs are described in the relevant sections.

\section{CRISPR/Cas9 Genome Editing}

CRISPR/Cas9 gRNAs were designed using using zifit.partners.org. The oligos 5' ACACCGCATCAACACTGGCACGAGCG 3' and AAAACGCTCGTGCCAGTGTTGATGCG 3' were annealed and cloned into the vector MLM3636 (Addgene \#43860) cut with BsmBI. The CRISPR gRNA plasmid was transfected with the JDS246 (Addgene plasmid \#43861) a mammalian codon optimized Cas9 nuclease with C-term 3X FLAG, using Genejuice transfection reagent (Merck) according to the manufacturer's instructions. Clonal cell lines were isolated by limiting dilution. Genomic DNA was isolated using DirectPCR lysis buffer (Viagen). Gene disruption was analysed by PCR using primers spanning the CRISPR gRNA binding region Sense 5'- aaagtctgttgcttgtgtttca - 3' and Antisense 5' taccagaatttgtagactgcgt- 3' and a PCR control region downstream of the CRISPR gRNA binding site to check quality of the genomic DNA preparations. Sequencing reactions were performed using the custom primer 5' tgacaactaaagcaccgcac 3 '.

\section{TALEN Genome Editing}

TALENS pairs were designed using the algorithm on zifit.partners.org. TALE repeats were created by nested PCR and cloned into the Fokl containing plasmids 
JDS71 (Addgene \#32285) for the Left TALEN (binding to the DNA sequence TCCTGTTTCAGCATCAAC) and JDS70 (Addgene \#32285) for the Right TALEN (binding to the DNA sequence TTCACCAGTATAAAGAAA). The TALEN pair is predicted to create a DNA double strand after the base pair 145 within exon 1 of the XIAP gene, corresponding to amino acid Arginine 48. TALEN transfections were performed using the Amaxa nucleofector, using the nucleofector kit $\mathrm{V}$ using the X-001 program. Clonal cell lines were isolated by limiting dilution and characterized using the same primer pairs used for the CRISPR/ Cas9 lines.

\section{Wound healing assay}

Cells were seeded onto 6 well plates at a density of 25000 cells/well and incubated at $37^{\circ} \mathrm{C}$ for 72 hours. A scratch was made across the well using a sterile pipette tip and cultured in fresh media. A line was drawn perpendicular to the scratch and an image was taken of the scratch underneath the line using an EVOS XL Core microscope. Plates were marked perpendicular to the scratch to ensure the same area was imaged at each time point. The wounds were imaged at 0, 6, 24 and 28 hours after the scratch was made. The area of the wound was measured at each time point using ImageJ software and normalised to $100 \%$ at time 0 . The data were plotted using GraphPad PRISM and the area underneath the curve was quantified. Each data point represents the area calculated from the closure of a single scratch. Statistical analysis was performed by Student's T-test or one-way ANOVA with Dunnett's multiple comparisons test. Where specified, the area under the curve was calculated and analysed using this method. A value of $p<0.05$ was considered to be statistically significant.

\section{Cell viability studies:}

Cells were seeded into 96 well plates at a density of 5000 cells/well and incubated at $37^{\circ} \mathrm{C}$ overnight. Etoposide (Sigma) or doxorubicin (Sigma) was diluted in supplemented DMEM to achieve a gradient of drug concentrations as specified in the figure legend. This was incubated at $37^{\circ} \mathrm{C}$ for 48 hours, after which the media was removed and $100 \mu \mathrm{l}$ of $5 \%$ PrestoBlue ${ }^{\circledR}$ (Invitrogen) diluted in supplemented 
DMEM was pipetted into each well. After 2 hours' incubation at $37^{\circ} \mathrm{C}, 90 \mu \mathrm{l}$ from each well was pipetted into a new 96 well plate and the fluorescence intensity was recorded at $570 \mathrm{~nm}$ using POLAR star Omega plate reader. The cell viability was expressed as a percentage relative to untreated controls.

\section{Immunoblotting and antibodies}

Immunoblot analysis was performed as described in ${ }^{37}$. Briefly, cell lysates were prepared with modified RIPA lysis buffer (50 mM Tris pH 8.0, $150 \mathrm{mM} \mathrm{NaCl}, 1 \%$ NP40, $0.5 \% \mathrm{C}_{24} \mathrm{H}_{39} \mathrm{NaO}_{4}, 0.1 \%$ SDS) supplemented with protease inhibitors and incubated on ice for $15 \mathrm{~min}$. Lysates were then sonicated in a Biodisrupter UltraSonic Waterbath (Diagenode) $(2 \times 30 \mathrm{~s}$ cycles on high) to shear the genomic DNA and clarified by centrifugation at $13000 \mathrm{rpm}$ at $4^{\circ} \mathrm{C}$ for $10 \mathrm{~min}$. Protein concentration was determined by BCA assay and $20 \mu \mathrm{g}$ of protein was loaded per well. Immunoblotting was performed using the following antibodies: $\beta$-actin (AC74, Sigma), XIAP (610762, BD Transduction Laboratories), XIAP (ab28151, Abcam), clAP1 (1E1-1-10, Enzo Life Sciences), C-RAF (A301-519A, Bethyl Laboratories) Rac1/Cdc42 (\#4651, Cell Signalling Technologies) and Actin (Ac74, Sigma).

\section{Caspase activity assay}

Cells were seeded into $96-$ well plates at a density of 5000 cells/well. The following day cells were treated as indicated and the caspase activity was determined using Caspase-Glo assay (Promega) according to the manufacturer's protocol. Raw RLU values are presented and statistical analysis performed using a one-way ANOVA with Dunnett's multiple comparisons test. A value of $p<0.05$ was considered to be statistically significant.

\section{Proliferation experiment}

Clonal cell lines were seeded into 96 well plates at a density of 2500 cells/well. The plates were incubated at $37^{\circ} \mathrm{C}$ and measured using $5 \%$ PrestoBlue ${ }^{8}$ (as described above) after either $24,48,72$ or 96 hours incubation. The gain was 
determined after 24 hours and was subsequently used to measure all plates to allow for comparison between time points. Raw values were plotted to indicate cell growth/ proliferation over time.

\section{SiRNA transfection}

siRNA duplex oligonucleotides were synthesised by MWG and transfected using Interferin (Polyplus) according to the manufacturer's instructions. SiRNA sequences: control-CAG UCG CGU UUG CGA CUG G; XIAP —GUG GUA GUC CUG UUU CAG C.

\section{Statistical analysis}

Statistical analysis was performed by a one-way ANOVA with Dunnett's multiple comparisons test or unpaired Student's t-test where appropriate. Where specified, the area under the curve was calculated and analysed using these methods. If a $p$-value is less than 0.05 , it is flagged with one star $\left(^{*}\right)$. If a $p$-value is less than 0.01 , it is flagged with two stars $\left({ }^{* *}\right)$. If a $p$-value is less than 0.001 , it is flagged with three stars $\left(^{* * *}\right)$. 


\section{RESULTS}

\section{Creation of XIAP-null cell lines}

To disrupt the coding region of the XIAP gene a CRISPR guide RNA (gRNA) was designed to bind within the first exon of the XIAP gene (Figure 1A). Plasmids encoding the CRISPR gRNA and the Cas9 nuclease were transfected into U2OS cells to induce DNA double strand breaks (DSBs) and disrupt the XIAP coding region (Figure $1 \mathrm{~A}$ ). DBSs were induced 150bp downstream from the start site of translation, corresponding to glycine 50 , within the first BIR of the XIAP protein (Figure $1 \mathrm{~A}$ ). PCR analysis was performed on genomic DNA isolated from clonal cell lines using specific primers flanking the site of DSBs to assess targeted gene disruption (Figure $1 \mathrm{~A}$ and $1 \mathrm{~B}$ ). Only clones with a single PCR product, indicating homozygosity, were taken forward for further analysis. Three disrupted clones were identified; two with PCR products smaller than the wildtype (small deletion of the XIAP gene) (KO 1 and KO 2) and one where the PCR product was absent (large deletion of the XIAP gene) (KO 3) (Figure 1B). The absence of PCR product from KO 3 was not due to lack of genomic DNA in the reaction, as control PCR primers amplifying an unrelated region of the XIAP coding sequence was efficiently amplified (Figure 1B). Rather it was predicted that a primer binding site was deleted from exon 1. DNA sequencing analysis revealed a $14 \mathrm{bp}$ deletion in clone $\mathrm{KO} 1$ and a $50 \mathrm{bp}$ deletion in clone $\mathrm{KO} 2$, which both result in frameshift mutations within the first exon of the XIAP gene (Figure 1C). Immunoblot analysis, using 2 different anti XIAP monoclonal antibodies, confirmed XIAP deletion in clones KO 1 and KO 2 and revealed that the large deletion of the XIAP gene in clone KO 3 also resulted in the creation of a XIAP-null cell line (Figure 1D and 1E). Together these results confirm 3 distinct genetic disruptions of the XIAP gene, allowing us to assess the effects of XIAP deletion in cells.

\section{XIAP null cells do not have increased sensitivity to cell death}

As XIAP is best described as a suppressor of caspase-dependent cell death we initially examined if XIAP-null U2OS cells are more sensitive to genotoxic stress induced by DNA damaging agents. Two wildtype and three independent isogenic 
XIAP-null cell lines were treated with increasing doses of the DNA topoisomerase II inhibitor, etoposide, or the DNA intercalating agent, doxorubicin, both potent inducers of apoptotic cell death. Depletion of XIAP levels by RNAi has previously been reported to increase caspase-dependent apoptotic cell death in response to these DNA damage inducing agents in cancer cell lines ${ }^{38}$. Both etoposide and doxorubicin both efficiently induced cell death in U2OS cells (Figure 2A and 2B, Supplemental Figure 1). Surprisingly, no differences in sensitivity were observed that correlated with XIAP status of the cell lines (Figure 2A and 2B, Supplementary Figure 1). Indeed, the IC50 values calculated for both etoposide and doxorubicin showed no significant differences between any of the XIAP-wildtype or XIAP-null cell lines tested (Figure 2C and 2D).

Our results indicate no differences in cell viability associated with XIAP status. However, cell viability assays do not specifically measure caspase activity associated with apoptotic cell death, but can in fact be influenced by the cumulative effects of alternative cell death pathways, cell growth rates and cell metabolic activity. As XIAP's role in cell death control is through its ability to bind to, and inhibit, the activity of cell death effector caspases we directly measured the activities of caspases -3 and -7 in XIAP-null cell lines in response to etoposide. XIAP wildtype and XIAP-null U2OS cells were treated with etoposide and apoptotic cell death was determined using a luminescent assay directly measuring caspase3 and caspase-7 activity (Figure 2E). Consistent with cell viability results, no significant differences in caspase activity in response to etoposide were observed between XIAP wildtype and null U2OS cell lines, indicating that loss of XIAP is not sufficient to alter either cell death or caspase activity in U2OS cells (Figure 2E). Together our data suggests that loss of XIAP has no effect on apoptotic cell death in response to DNA damaging agents in our genetically modified cell lines.

\section{XIAP-null cells have increased migratory capacity}

XIAP has the ability to act as an E3 ubiquitin ligase and as such is an important regulator of several cell signaling pathways ${ }^{10,28,39}$. Several studies have identified IAP proteins, including XIAP, as regulators of the cytoskeleton ${ }^{28}$. 
However, there have been conflicting reports on the specific role of XIAP in regulating cell motility. There is data suggesting that elevated XIAP promotes cell motility ${ }^{23,26}$ and other reports suggesting that high XIAP suppresses migration rates in cultured cell lines ${ }^{24,25}$. We therefore decided to use XIAP-null cells to investigate if genetic deletion of the XIAP gene alters migration rates in U2OS cells.

XIAP wildtype and null U2OS cell lines were seeded and allowed to grow to confluency before a scratch was created using a sterile pipette tip. Multiple images of the scratch were taken over the course of the assay and area was mapped and measured using ImageJ software. The data indicate that genetic deletion of XIAP increased the migration rates, as indicated by the enhanced closure rates of the wounds in XIAP-null cells (Figure 3A and Supplementary Figure 2A). To quantify the migratory capacity of each clonal cell line area under the curve was calculated to map wound closure rates and XIAP deletion resulted in a significantly increased cell migration rate in each of the cell lines tested (Figure 3B and $C$ ). To ensure that the increased closure of the wound healing assays in XIAP-null cells was indeed due to increased cell motility, and not increased cell growth/ doubling time, cell proliferation rates were compared between wild type and XIAP-null cells (Figure 3D). No differences were seen in growth rates between wildtype and XIAPnull cells indicating that differences in wound closure were indeed due to differences in cell motility rather than an increase in cell number (Figure 3D). Indeed, quantitative and qualitative analysis showed increased morphological changes and motility, in XIAP-null cells by measuring the dynamic nature of the migration front (Supplementary Figure $2 \mathrm{~B}$ and $2 \mathrm{C}$ ).

Like all genome editing technologies CRISPR/Cas9 can induce double strand breaks at off-target sites within the genomes of targeted cells ${ }^{40}$. To ensure the XIAP-dependent migration phenotype was in fact due to the targeted disruption of the XIAP gene, rather than an off-target effect of the CRISPR gRNA, we tested an independent XIAP-null cell line created using transcription activator-like effector nucleases (TALENs), an alternative genome editing tool. Plasmids encoding a TALEN pair were designed to induce a double strand break within the first exon of 
XIAP, 145 bp downstream of the start site of translation corresponding to arginine 48 of the XIAP protein (Supplementary Figure $3 \mathrm{~A}$ ). Clonal cell lines were isolated by limiting dilutions and analysed by PCR (Supp. Figure 3B). A clone homozygous for a deletion in the XIAP coding sequence was identified (Supplementary Figure 3B). Sequencing analysis confirmed a 10bp deletion of the XIAP coding sequence, predicted to result in a frame shift and premature stop codon (Supplementary Figure 3 C). XIAP deletion was confirmed by immunoblot analysis (Supp. Figure 3D). Cell motility was measured using the TALEN-XIAP null cell line and compared to a matched wildtype control (Supplementary Figure 3E). TALENmediated XIAP deletion resulted in extended morphology and increased cell motility, consistent with the results observed in the CRISPR/ Cas9 XIAP-null cell lines (Supplementary Figure 3D and 3E). To investigate if our results were cell line specific we next tested the migratory capacity of HeLa cells in which XIAP is depleted by RNAi-mediated knockdown to investigate if our effects were observed across cell lines (Supplementary Figure 4A). Consistent with results from genetically engineered cells XIAP depletion resulted in increased migratory capacity as measured by wound healing assay (Supplementary Figure 4B and 4C). Together our results indicate that XIAP deletion increases the migratory capacity of culture cells independently of the method used to create them.

\section{cIAP depletion does not significantly alter cell migration rates in U2OS cells}

The related cellular IAP (CIAP) proteins, cIAP1 and clAP2, have also been implicated in altering cell migration rates in cultured cell lines ${ }^{23,26}$. As deletion of IAP genes can influence the protein levels of other family members through feedback mechanisms we analysed cIAP levels in XIAP-null cell lines ${ }^{41}$. A modest, but insignificant, increase in clAP1 is observed in XIAP null cells consistent with the feedback loop observed in other cellular systems (Figure 4A, Supplementary Figure 5A). To examine the potential roles of clAP proteins in cell migration, independently from XIAP, U2OS wildtype cells were treated with the SMAC mimetic compound, LCL $161^{42}$. SMAC mimetics, such as LCL 161, although designed to bind to XIAP have been shown to target both CIAP1 and 
cIAP2 for proteasomal mediated degradation, without altering XIAP levels ${ }^{43,44}$. Indeed, immunoblot analysis of U2OS cell lysates prepared from cells treated with LCL161 revealed a rapid and specific reduction of CIAP1, which was observed as quickly as $30 \mathrm{~min}$ after treatment and persisted for the course of the migration experiment (Figure 4B). As we could suppress cIAP1 levels, without altering XIAP, we measured cell migration rates in LCL161 treated cells. Cell migration was not significantly altered in cells treated with LCL161 (Figure 4C). The data suggest that chemical depletion of cIAP proteins does not alter cell migration rates in U2OS cells.

\section{XIAP E3 ubiquitin Ligase Activity is Required to Suppress Cell Migration}

The XIAP protein has dual enzymatic activities, by virtue of its ability to bind caspases via its BIR domains and its E3 ubiquitin ligase activity conferred by the C-terminal RING domain (Figure 5A). To investigate what properties of XIAP are necessary for the control of cell migration in U2OS cells, wildtype XIAP and XIAP variants were transfected into XIAP null cell lines. Based on the crystal structures of XIAP, specific point mutations have been characterized that can alter specific properties of the protein ${ }^{5,6}$. To examine the properties of XIAP required for its ability to regulate cell migration we reconstituted XIAP null cells with wildtype XIAP,

a caspase $3 / 7 / 9$ binding mutant (D148A/ W310A) ${ }^{45,46}$ or an E3 ubiquitin ligase mutant (F495A) ${ }^{47}$ (Figure 5A). Immunoblot analysis revealed that each XIAP variant was expressed to equivalent levels in XIAP-null cells (Figure 5B). Cells reconstituted with wildtype XIAP or the caspase-binding mutant (D148A/ W310A), significantly inhibited the migration of XIAP deficient cells as compared to the XIAP-deleted control cell line transfected with empty vector (Figure $5 \mathrm{C}$ and $5 \mathrm{D}$ ). However, reconstitution with the XIAP ubiquitin ligase mutant, F495A, failed to reverse the increased migration phenotype, indicating that the ubiquitin ligase activity of XIAP is essential for its ability to regulate cell motility in cells (Figure 5C and 5D).

\section{XIAP regulates levels of the C-RAF protein kinase to regulate cell motility}


Previous studies have shown that XIAP can suppress cell migration by binding to and ubiquitinating C-RAF and Cdc42, key proteins involved in cell motility, marking then for proteasomal degradation. ${ }^{48}{ }^{26}$. C-RAF, a serine/threonine kinase in the MAP kinase signaling cascade, is a well-described promotor of cell migration and motility, and Cdc42 is a RhoGTPase that controls filopodia formation and cell migration ${ }^{48} 23,26$. As our data suggested that XIAP suppresses migration rates we examined levels of C-RAF and Cdc42 in the XIAP null cells. Immunoblot analysis of cell lysates prepared from XIAP wildtype and XIAP null cell lines indicate that XIAP null cells have elevated levels of C-RAF protein, but not elevated levels of $\mathrm{Cdc} 42$ (Figure 6A and 6B, Supplementary Figure $5 B$ and $C$ ). To investigate if expression of XIAP could reduce C-RAF levels, XIAP null cells were transfected with plasmids encoding wildtype XIAP or empty vector. Expression of XIAP reduced levels of C-RAF compared those observed in XIAP null cells (Figure 6C, Supplementary 5C). Our data indicate that C-RAF levels, but not Cdc42, are elevated in XIAP-null cells, and C-RAF levels can be reduced by re-introducing wildtype XIAP.

Therefore, we decided to investigate the role of C-RAF activity in regulating the rates of migration and motility. To suppress C-RAF activity XIAP-null cells were treated with the C-RAF kinase inhibitor, BAY 43-9006 ${ }^{49}$. XIAP wildtype and knockout cells were pretreated BAY 43-9006, and cell migration rates were determined by wound healing assay compared to cells treated with vehicle alone. The migration rates of XIAP-null cells was significantly suppressed by the C-RAF inhibitor, indicating that C-RAF levels and activity were at least in part responsible for the increased migration rates in the XIAP-null cells (Figure 6D).

Taken together these results indicate that XIAP deletion results in enhanced cellular migration rates. We find that it is the ubiquitin ligase activity of XIAP is important for the migration phenotype, through regulation of the levels of the CRAF protein kinase. In addition, we show that chemical inhibition of C-RAF reverses the enhanced cell migration observed in XIAP-null cells. 


\section{Discussion}

The results of the present study indicate that XIAP plays an important role in controlling cell migration rates. Our data, using CRISPR/Cas9 and TALEN modified cell lines demonstrate that XIAP deletion increases the motility of U2OS cells, by controlling the levels of the C-RAF protein kinase. Reconstitution of our XIAP-null cell lines with wildtype XIAP, and XIAP variants, suppress C-RAF levels and reduce cell motility, in a manner dependent on XIAP ubiquitin ligase activity. C-RAF protein levels are elevated in XIAP-null cells and reduced when XIAP is reexpressed. Importantly, treatment of XIAP-null cells with a C-RAF kinase inhibitor is sufficient to supress the increased cell migration rates.

XIAP is a well-described inhibitor of caspase-3, -7 and -9 activity, with overexpression of the XIAP protein a well-defined suppressor of cell death 5,7,29,39. As such a number of drugs that directly target XIAP are in development and these inhibitors might enhance chemosensitivity in XIAP over-expressing tumours ${ }^{50-52}$. However, the cellular role of endogenous XIAP in the regulation apoptotic cell death is less clear. The XIAP-null mouse is born at expected Mendalian ratios with no obvious cell death related phenotype ${ }^{53}$. Instead, many studies have indicated that a key role for the IAP family of proteins is to act as E3 ubiquitin ligases to regulate multiple cellular processes. Indeed, XIAP deficiency can result in more aggressive disease in a murine prostate cancer model which suggests a degree of caution to be employed before XIAP antagonists are used for cancer therapy ${ }^{54}$.

Several studies have previously demonstrated a link between XIAP and CIAP levels and cell motility/ migration ${ }^{28}$. XIAP deletion or depletion has been reported to increase cell migration and motility through increasing levels of C-RAF and Cdc42 2326 . However, conflicting reports have demonstrated that XIAP can negatively regulate RhoGDI activity by modulating its SUMOylation, thereby increasing actin polymerization and cell motility, resulting in XIAP depletion

causing decreases in cell migration 245556 . These conflicting results may arise by localised differences of the downstream IAP signalling molecules in each individual cell type. Results from cell lines used our study show that XIAP deletion increases 
levels of C-RAF, and C-RAF inhibition reduced the migratory capacity of XIAP deficient cells lines.

Our data show that XIAP dependent control of cell migration is at least in part due to its ability to modulate the levels of the C-RAF protein kinase. A major challenge in understanding XIAP-deficient disease is reconciling the cellular properties of XIAP with the symptoms associated disease. One of the major symptoms associated with XIAP deficiency is the development of severe haemorrhagic colitis, which is associated with its key role in inflammatory signalling through the NOD signalling pathway ${ }^{57-60}$. However, XIAP-deficient patients also present with hemophagocytic lymphohistiocytosis (HLH), recurrent fevers, recurrent low blood counts and splenomegaly, which may be due to XIAP's role in regulating caspases or alternative signalling pathways ${ }^{61}$. Interestingly, in the context of the current study, it has been reported that constitutively active mitochondrial C-RAF can also result in mild to moderate splenomegaly ${ }^{62}$. Indeed, expression of a kinase-activating C-RAF mutant, C-RAF(L613V), in a murine model of Noonan syndrome, results in splenomegaly, a phenotype also associated with XIAP-deficient patients ${ }^{63}$. It would be interesting to investigate if the regulation of C-RAF and cell migration by XIAP has any role in the progression of XLP2, either through spleen enlargement or progression of any of the other phenotypes.

Collectively our data reveal that XIAP has a critical role in regulating cell migration through its ability to polyubiquitinate and target C-RAF for proteolytic destruction. These results could provide further clues to the role of XIAP in human disease. 


\section{Acknowledgements}

We would like to thank Professor Colin Duckett for his supply of reagents. We would also like to thank Dr Iglika Ivanova and Dr Adrian Yemm for helpful discussions and reviewing drafts of the manuscript. Funding for this work was provided by Newcastle University Independent Researcher Establishment Scheme Award (to N.S.K.) and Cancer Research UK [C1443/A22095 to J.E.H];: Funding for open access charge: Newcastle University. 


\section{Author contributions}

LGR and NSK designed the research. LGR, LAKD, JEH and NSK performed the research. All authors analysed the data. NSK wrote the manuscript.

Competing Interests

The authors declare no competing interests. 
References

1. Srinivasula, S. M. \& Ashwell, J. D. IAPs: What's in a Name? Molecular Cell (2008) doi:10.1016/j.molcel.2008.03.008.

2. Crook, N. E., Clem, R. J. \& Miller, L. K. An apoptosis-inhibiting baculovirus gene with a zinc finger-like motif. J. Virol. (1993).

3. Gyrd-Hansen, M. \& Meier, P. IAPs: From caspase inhibitors to modulators of NF-KB, inflammation and cancer. Nature Reviews Cancer (2010) doi:10.1038/nrc2889.

4. Latour, S. \& Aguilar, C. XIAP deficiency syndrome in humans. Seminars in Cell and Developmental Biology (2015) doi:10.1016/j.semcdb.2015.01.015.

5. Riedl, S. J. et al. Structural basis for the inhibition of caspase-3 by XIAP. Cell (2001) doi:10.1016/S0092-8674(01)00274-4.

6. Chai, J. et al. Structural basis of caspase-7 inhibition by XIAP. Cell (2001) doi:10.1016/S0092-8674(01)00272-0.

7. Shiozaki EN, Chai J, Rigotti DJ, Riedl SJ, Li P, Srinivasula SM, Alnemri ES, Fairman R, S. Y. Mechanism of XIAP-mediated inhibition of caspase-9. Mol. Cell (2003) doi:10.1016/S1097-2765(03)00054-6.

8. Duckett1I, C. S. et al. A conserved family of cellular genes related to the baculovirus iap gene and encoding apoptosis inhibitors. EMBO J. (1996).

9. Liston, P. et al. Suppression of apoptosis in mammalian cells by NAIP and a related family of IAP genes. Nature (1996) doi:10.1038/379349a0.

10. Galbán, S. \& Duckett, C. S. XIAP as a ubiquitin ligase in cellular signaling. Cell Death and Differentiation (2010) doi:10.1038/cdd.2009.81.

11. Yang, Y., Fang, S., Jensen, J. P., Weissman, A. M. \& Ashwell, J. D. Ubiquitin protein ligase activity of IAPs and their degradation in proteasomes in response to apoptotic stimuli. Science (80-. ). (2000) doi:10.1126/science.288.5467.874.

12. Wu, Z. H. et al. ATM- and NEMO-dependent ELKS ubiquitination coordinates TAK1-Mediated IKK activation in response to genotoxic stress. Mol. Cell (2010) doi:10.1016/j.molcel.2010.09.010. 
13. Jin, H. S. et al. clAP1, clAP2, and XIAP act cooperatively via nonredundant pathways to regulate genotoxic stress-induced nuclear factor-kB activation. Cancer Res. (2009) doi:10.1158/0008-5472.CAN-08-2256.

14. Sanna, M. G., Duckett, C. S., Richter, B. W. M., Thompson, C. B. \& Ulevitch, R. J. Selective activation of JNK1 is necessary for the antiapoptotic activity of hILP. Proc. Natl. Acad. Sci. (1998) doi:10.1073/pnas.95.11.6015.

15. Mufti, A. R. et al. XIAP is a copper binding protein deregulated in Wilson's disease and other copper toxicosis disorders. Mol. Cell (2006) doi:10.1016/j.molcel.2006.01.033.

16. Brady, G. F. et al. Regulation of the Copper Chaperone CCS by XIAPMediated Ubiquitination. Mol. Cell. Biol. (2010) doi:Doi 10.1128/Mcb.00900-09.

17. Burstein, E. et al. A novel role for XIAP in copper homeostasis through regulation of MURR1. EMBO J. (2004) doi:10.1038/sj.emboj.7600031.

18. Park, C. V., Ivanova, I. G. \& Kenneth, N. S. XIAP upregulates expression of HIF target genes by targeting HIF1a for Lys63-linked polyubiquitination. Nucleic Acids Res. (2017) doi:10.1093/nar/gkx549.

19. Hanson, A. J. et al. XIAP Monoubiquitylates Groucho/TLE to Promote Canonical Wnt Signaling. Mol. Cell (2012) doi:10.1016/j.molcel.2011.12.032.

20. Huang, X., Wu, Z., Mei, Y. \& Wu, M. XIAP inhibits autophagy via XIAPMdm2-p53 signalling. EMBO J. (2013) doi:10.1038/emboj.2013.133.

21. Lin, F. et al. XIAP and cIAP1 amplifications induce Beclin 1-dependent autophagy through NFKB activation. Hum. Mol. Genet. (2015) doi:10.1093/hmg/ddv052.

22. Gradzka, S. et al. Inhibitor of apoptosis proteins are required for effective fusion of autophagosomes with lysosomes article. Cell Death Dis. (2018) doi:10.1038/s41419-018-0508-y.

23. Oberoi, T. K. et al. IAPs regulate the plasticity of cell migration by directly targeting Rac1 for degradation. EMBO J. (2012) 
doi:10.1038/emboj.2011.423.

24. Liu, J. et al. X-linked Inhibitor of Apoptosis Protein (XIAP) mediates cancer cell motility via Rho GDP Dissociation Inhibitor (RhoGDI)-dependent regulation of the cytoskeleton. J. Biol. Chem. (2011) doi:10.1074/jbc.M110.176982.

25. Liu, J. et al. E3 ligase activity of XIAP RING domain is required for XIAPmediated cancer cell migration, but not for its RhoGDI binding activity. PLoS One (2012) doi:10.1371/journal.pone.0035682.

26. Dogan, T. et al. X-linked and cellular IAPs modulate the stability of C-RAF kinase and cell motility. Nat. Cell Biol. (2008) doi:10.1038/ncb1804.

27. To, K. K. W. \& Huang, L. E. Suppression of hypoxia-inducible factor $1 \alpha$ (HIF-1 $\alpha$ ) transcriptional activity by the HIF prolyl hydroxylase EGLN1. J. Biol. Chem. (2005) doi:10.1074/jbc.M504342200.

28. Kenneth, N. S. \& Duckett, C. S. IAP proteins: Regulators of cell migration and development. Current Opinion in Cell Biology (2012) doi:10.1016/j.ceb.2012.11.004.

29. Zhang, Y. et al. X-linked inhibitor of apoptosis positive nuclear labeling: A new independent prognostic biomarker of breast invasive ductal carcinoma. Diagn. Pathol. (2011) doi:10.1186/1746-1596-6-49.

30. Mizutani, Y. et al. Overexpression of XIAP expression in renal cell carcinoma predicts a worse prognosis. Int. J. Oncol. (2007).

31. Ramp, U. et al. XIAP expression is an independent prognostic marker in clear-cell renal carcinomas. Hum. Pathol. (2004) doi:10.1016/j.humpath.2004.03.011.

32. Li, M., Song, T., Yin, Z. F. \& Na, Y. Q. XIAP as a prognostic marker of early recurrence of nonmuscular invasive bladder cancer. Chin. Med. J. (Engl). (2007).

33. Tamm, I. et al. Expression and prognostic significance of IAP-family genes in human cancers and myeloid leukemias. Clin. Cancer Res. (2000).

34. Li, S. et al. Prognostic significance of X-linked inhibitor of apoptosis protein in solid tumors: A systematic review and meta-analysis. J. Cell. Physiol. $\mathbf{0}$, 
(2019).

35. Rigaud, S. et al. XIAP deficiency in humans causes an X-linked lymphoproliferative syndrome. Nature (2006) doi:10.1038/nature05257.

36. Mudde, A. C. A., Booth, C. \& Marsh, R. A. Evolution of Our Understanding of XIAP Deficiency. Frontiers in Pediatrics (2021) doi:10.3389/fped.2021.660520.

37. Ivanova, I. G., Park, C. V, Yemm, A. I. \& Kenneth, N. S. PERK/eIF2a signaling inhibits HIF-induced gene expression during the unfolded protein response via YB1-dependent regulation of HIF1a translation. Nucleic Acids Res. 46, 3878-3890 (2018).

38. Lima, R. T., Martins, L. M., Guimarães, J. E., Sambade, C. \& Vasconcelos, M. H. Specific downregulation of bcl-2 and XIAP by RNAi enhances the effects of chemotherapeutic agents in MCF-7 human breast cancer cells. Cancer Gene Ther. (2004) doi:10.1038/sj.cgt.7700706.

39. Yang, Y. L. \& Li, X. M. The IAP family: Endogenous caspase inhibitors with multiple biological activities. Cell Research (2000) doi:10.1038/sj.cr.7290046.

40. Gaj, T., Gersbach, C. A. \& Barbas, C. F. ZFN, TALEN, and CRISPR/Casbased methods for genome engineering. Trends in Biotechnology (2013) doi:10.1016/j.tibtech.2013.04.004.

41. Heard, K. N., Bertrand, M. J. \& Barker, P. A. clAP2 supports viability of mice lacking cIAP1 and XIAP. EMBO J. (2015) doi:10.15252/embj.201592060.

42. Weisberg, E. et al. Smac mimetics: Implications for enhancement of targeted therapies in leukemia. Leukemia (2010) doi:10.1038/leu.2010.212.

43. Somasekharan, S. P. et al. YB-1 regulates stress granule formation and tumor progression by translationally activating G3BP1. J. Cell Biol. (2015) doi:10.1083/jcb.201411047.

44. Yang, C. et al. LCL161 increases paclitaxel-induced apoptosis by degrading cIAP1 and cIAP2 in NSCLC. J. Exp. Clin. Cancer Res. (2016) doi:10.1186/s13046-016-0435-7. 
45. Bratton, S. B., Lewis, J., Butterworth, M., Duckett, C. S. \& Cohen, G. M. XIAP inhibition of caspase-3 preserves its association with the Apaf-1 apoptosome and prevents CD95- and Bax-induced apoptosis. Cell Death Differ. (2002) doi:10.1038/sj.cdd.4401069.

46. Silke, J. et al. The anti-apoptotic activity of XIAP is retained upon mutation of both the caspase 3- and caspase 9-interacting sites. J. Cell Biol. (2002) doi:10.1083/jcb.200108085.

47. Gyrd-Hansen, M. et al. IAPs contain an evolutionarily conserved ubiquitinbinding domain that regulates NF-KB as well as cell survival and oncogenesis. Nat. Cell Biol. (2008) doi:10.1038/ncb1789.

48. Murali, A. et al. Ubiquitin-dependent regulation of Cdc42 by XIAP. Cell Death Dis. (2017) doi:10.1038/cddis.2017.305.

49. Wilhelm, S. M. et al. BAY 43-9006 exhibits broad spectrum oral antitumor activity and targets the RAF/MEK/ERK pathway and receptor tyrosine kinases involved in tumor progression and angiogenesis. Cancer Res. (2004) doi:10.1158/0008-5472.CAN-04-1443.

50. Nachmias, B., Ashhab, Y. \& Ben-Yehuda, D. The inhibitor of apoptosis protein family (IAPs): An emerging therapeutic target in cancer. Semin. Cancer Biol. (2004) doi:10.1016/j.semcancer.2004.04.002.

51. Vucic, D. \& Fairbrother, W. J. The inhibitor of apoptosis proteins as therapeutic targets in cancer. Clinical Cancer Research (2007) doi:10.1158/1078-0432.CCR-07-0729.

52. Kenneth, N. S., Mudie, S., Naron, S. \& Rocha, S. TfR1 interacts with the IKK complex and is involved in IKK-NF-KB signalling. Biochem. J. (2013) doi:10.1042/BJ20120625.

53. Harlin, H., Reffey, S. B., Duckett, C. S., Lindsten, T. \& Thompson, C. B. Characterization of XIAP-Deficient Mice. Mol. Cell. Biol. (2002) doi:10.1128/mcb.21.10.3604-3608.2001.

54. Hwang, C. et al. X-linked inhibitor of apoptosis deficiency in the TRAMP mouse prostate cancer model. Cell Death Differ. (2008) doi:10.1038/cdd.2008.15. 
55. Amininejad, L. et al. Analysis of Genes Associated With Monogenic

Primary Immunodeficiency Identifies Rare Variants in XIAP in Patients With Crohn's Disease. Gastroenterology (2018) doi:10.1053/j.gastro.2018.02.028.

56. $\mathrm{Yu}, \mathrm{J}$. et al. RhoGDI SUMOylation at Lys-138 increases its binding activity to Rho GTPase and its inhibiting cancer cell motility. J. Biol. Chem. (2012) doi:10.1074/jbc.M111.337469.

57. Krieg, A. et al. XIAP mediates NOD signaling via interaction with RIP2. Proc. Natl. Acad. Sci. (2009) doi:10.1073/pnas.0907131106.

58. Damgaard, R. B. et al. The Ubiquitin Ligase XIAP Recruits LUBAC for NOD2 Signaling in Inflammation and Innate Immunity. Mol. Cell (2012) doi:10.1016/j.molcel.2012.04.014.

59. Damgaard, R. B. et al. Disease-causing mutations in the XIAP BIR2 domain impair NOD2-dependent immune signalling. EMBO Mol. Med. (2013) doi:10.1002/emmm.201303090.

60. Crowley, E. et al. X-Linked Inhibitor of Apoptosis Protein (XIAP) Genetic Variants in Paediatric-Onset IBD. Gastroenterology (2017) doi:10.1016/s0016-5085(17)32905-0.

61. Filipovich, A. H. et al. Clinical similarities and differences of patients with Xlinked lymphoproliferative syndrome type 1 ( XLP-1 / SAP deficiency ) versus type 2 ( XLP-2 / XIAP deficiency ). Blood (2011) doi:10.1182/blood2010-07-298372.The.

62. Salomoni, P. et al. Expression of Constitutively Active Raf-1 in the Mitochondria Restores Antiapoptotic and Leukemogenic Potential of a Transformation-deficient BCR/ABL Mutant. J. Exp. Med. (2002) doi:10.1084/jem.187.12.1995.

63. Wu, X. et al. MEK-ERK pathway modulation ameliorates disease phenotypes in a mouse model of Noonan syndrome associated with the Raf1L613V mutation. J. Clin. Invest. (2011) doi:10.1172/JCI44929. 


\section{Figure Legends}

Figure 1 Creation of XIAP null cell lines. A. Schematic of the exon structure of the XIAP gene and the domains of the XIAP protein. Location of the CRISPR gRNA binding site and the diagnostic PCR primers are indicated. B. PCR analysis of genomic DNA prepared from U2OS clonal cell lines prepared from CRISPR/Cas9 transfected cells. C. Alignment of the sequencing data of XIAP exon 1 from clonal cells lines. Whole cell lysates were prepared from CRISPR/ Cas9 monoclonal U2OS cells. Lysates were subjected to immunoblot analysis to assess XIAP expression levels using D. an XIAP antibody from BD (immunogen amino acids 268-426) and E. an Abcam XIAP antibody (immunogen amino acids 352-449).

Figure 2 XIAP status does not alter cell viability in response to cytotoxic agents A. U2OS clonal cell lines were exposed to etoposide for 48 hours and viability was determined by PrestoBlue assay. The normalised mean fluorescence intensity $(\mathrm{MFI})$ at increasing concentrations of etoposide $(\log 2(\mathrm{mM}))$ of three independent experiments for each clone is averaged and plotted (Individual repeats shown in Supp Figure 1A). Statistical analysis performed using a one-way ANOVA with Dunnett's multiple comparisons test. B. As in A but using doxorubicin. C. The mean normalised IC50 $(\mathrm{mM})$ for etoposide is plotted for each clone. D. As in C. but for doxorubicin. E. U2OS clonal cell lines treated with $50 \mathrm{uM}$ Etoposide for $24 \mathrm{~h}$ as indicated. Caspase-3 -7 activity was determined by Caspase-Glo assay and values normalised to untreated controls. Error bars represent standard deviation. Statistical analysis performed using a one-way ANOVA with Dunnett's multiple comparisons test.

\section{Figure 3 XIAP-null cells have increased migratory capacity. A.} Representative images of a wound healing experiment for wild type and XIAP null clones at 0 and 24 hours. The migration front is highlighted for clarity. B. The area of the wound was imaged and measured at 0,8 , and 24 hours using Image J 
software. The data was normalised with the percentage wound area equalling $100 \%$ at 0 hours. The $\%$ wound area was plotted against time and the area under the curve (\% wound area $x$ time(hours)) was calculated and analysed by one-way ANOVA using Dunnett multiple comparisons test in comparison to the wildtype clone 1. Each measurement represents data from an individual scratch. Statistical analysis performed using a one-way ANOVA with Dunnett's multiple comparisons test C. As in B. using independent XIAP-null clonal cell lines. D. Proliferation assay performed using Prestoblue assay. Cell lines and measurement times as indicated.

Figure 4. cIAP proteins does not significantly alter U2OS cell motility. A. Whole cell lysates were prepared from CRISPR/ Cas9 monoclonal U2OS cells. Lysates were subjected to immunoblot analysis to assess clAP1 levels. B. Wildtype U2OS cells were treated with the SMAC mimetic compound, LCL161, for the indicated times. Whole cell lysates were prepared and analysed by immunoblot analysis using the indicated antibodies. C. Wound healing assays were performed on untreated and LCL161 treated U2OS cells and measured at 0 , 8 and 24 hours. The area of the wound was imaged and measured at 0,8 , and 24 hours using ImageJ software. The data was normalised with the percentage wound area equalling $100 \%$ at 0 hours. The \% wound area was plotted against time and the area under the curve (\% wound area $x$ time(hours)) was calculated and analysed by unpaired students t-test.

Figure 5. The ubiquitin ligase activity of XIAP is necessary to control cell migration rates. A. Schematic of XIAP protein indicating key residues essential for mediating the caspase interactions and the E3 ubiquitin ligase activity. B. Representative images of a wound healing experiment using XIAP KO clone 2 transfected with empty vector, wildtype XIAP, caspase binding mutant XIAP (D148A/ W310A), and ubiquitin ligase mutant XIAP (F495A), and XIAP WT clone 1 transfected with empty vector at 0 and 24 hours. The migration front is highlighted for clarity. C. Whole cell lysates prepared from transfected cells and 
immunoblotted with XIAP and actin antibodies to determine transfection efficiency. D. The area of the wound was imaged and measured at 0,8 , and 24 hours using ImageJ software. The data was normalised with the percentage wound area equalling $100 \%$ at 0 hours. The $\%$ wound area was plotted against time and the area under the curve (\% wound area $\mathrm{x}$ time(hours)) was calculated and analysed by one-way ANOVA using Dunnett multiple comparisons test in comparison to the XIAP KO clone 2 transfected with empty vector.

Figure 6. XIAP controls levels of C-RAF to influence cell migration. A,B. Whole cell lysates were prepared from CRISPR/ Cas9 monoclonal U2OS cells and immunoblotted using the indicated antibodies. C. The area of the wound of XIAP null cells (KO 2) treated with the C-RAF inhibitor, BAY 43-9006, was imaged and measured at 0, 8, and 24 hours using Image J software. The data was normalised with the percentage wound area equalling $100 \%$ at 0 hours. The $\%$ wound area was plotted against time and the area under the curve (\% wound area $x$ time(hours)) was calculated and analysed by unpaired students t-test. 
Figure 1

A

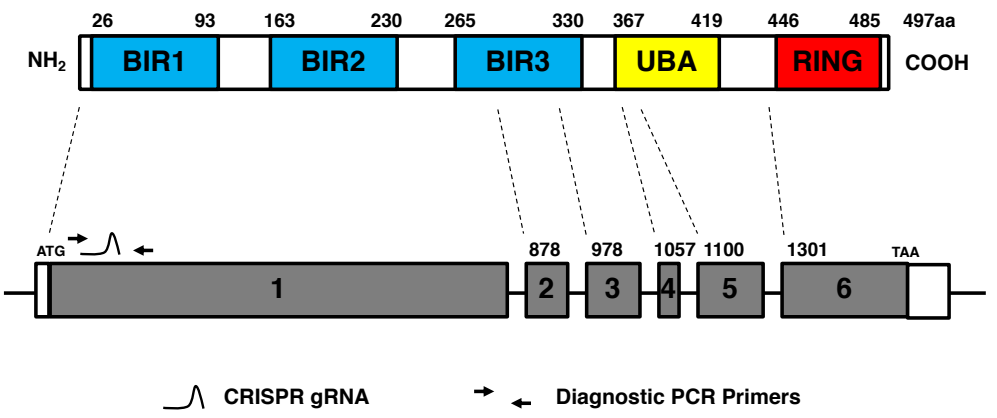

B

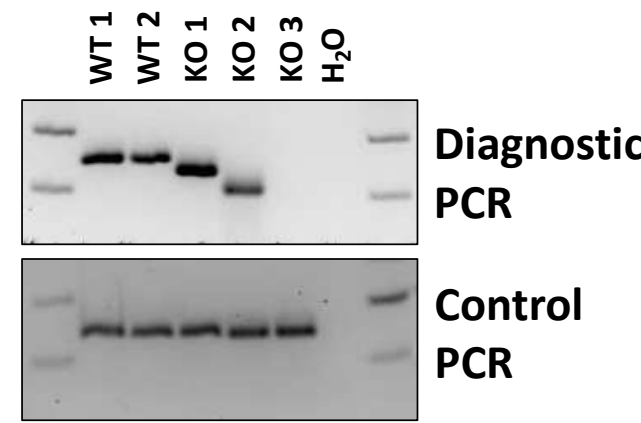

C

\section{CRISPR gRNA binding}

XIAP 91-aaacttttgctaattttccaagtggtagtcctgtttca GCATCAACACTGGCACGAGCaGGgtttctttatactggtgaa-170

WT 1 91-aaactttgctaatttccaagtggtagtcctgtttcagcatcaacactggcacgagcagggtttctttatactggtgaa-170

WT 2 91-aaactttgctaatttccaagtggtagtcctgtttcagcatcaacactggcacgagcagggtttctttatactggtgaa-170

Kо 1 91-aaacttt.gctaattt.ccaagtggtagtcctgtttcagcatcaacactggcacga--------------tactggtgaa-156

ко 2 91-aaact---120

ко 3.

D

$\begin{array}{lllll}-1 & N & r & N & m \\ 5 & ⺊ & 0 & 0 & 0\end{array}$

XIAP (BD)

actin

$\mathbf{E}$

$\begin{array}{lllll}-1 & N & r & N & m \\ ⿱ ⺊ & ⺊ & 0 & 0 & 0\end{array}$

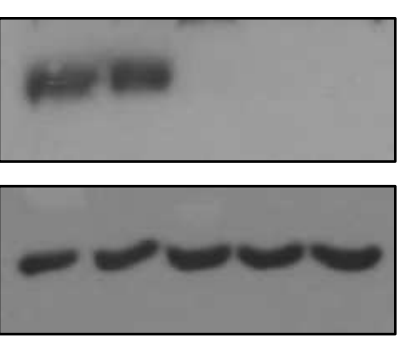

actin 
Figure 2

A

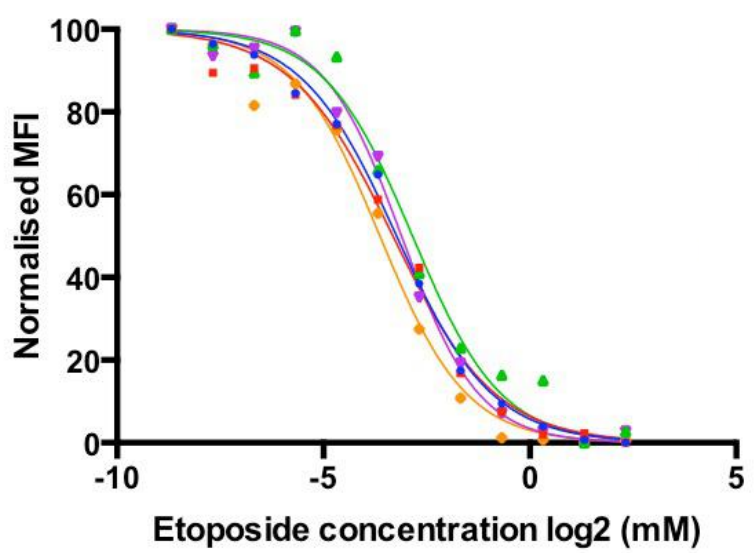

C

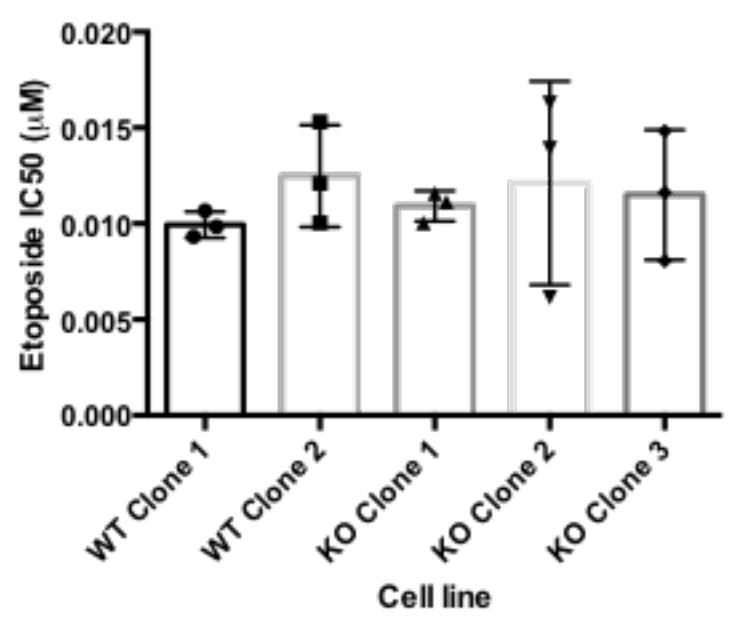

B

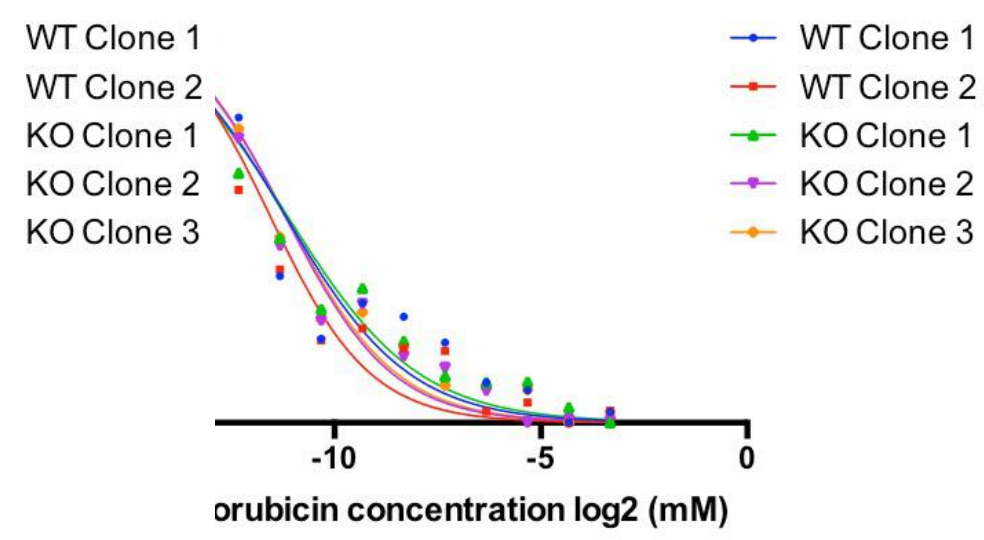

D

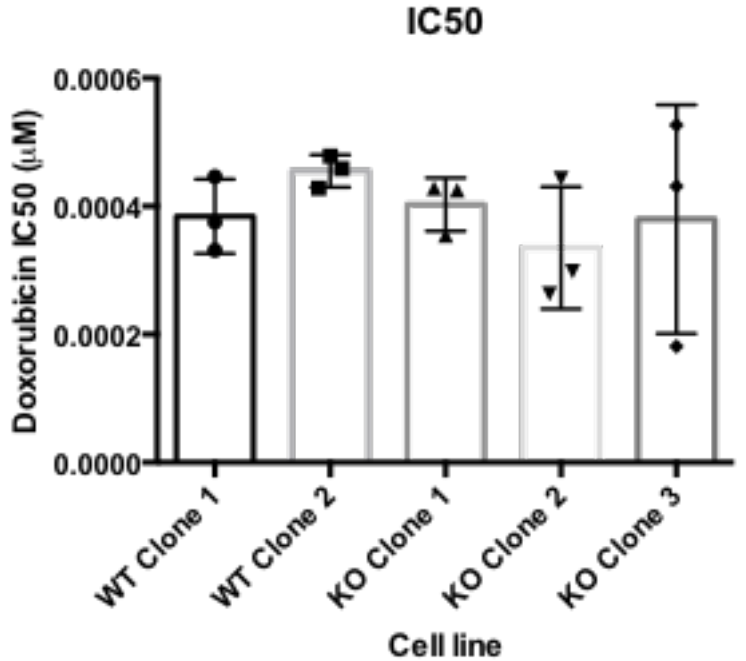

E

Caspase-3/ -7 Activity

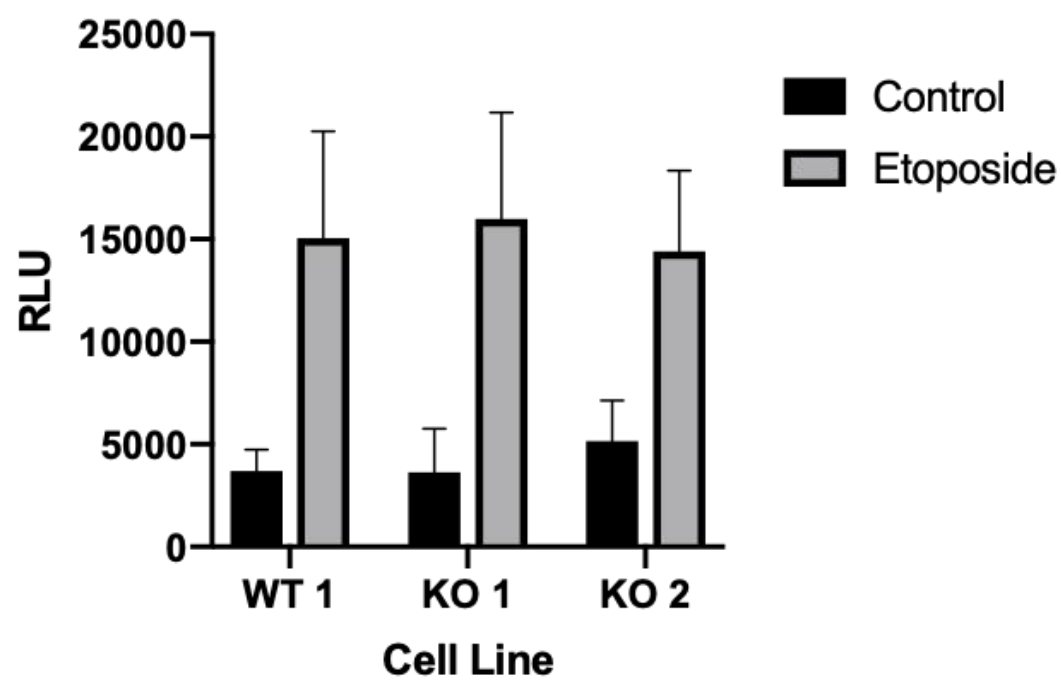


Figure 3

A

WT Clone 1

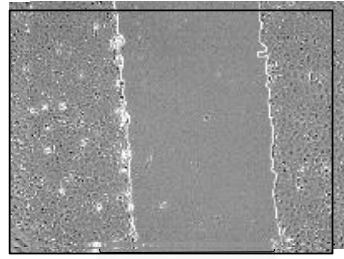

$24 \mathrm{~h}$

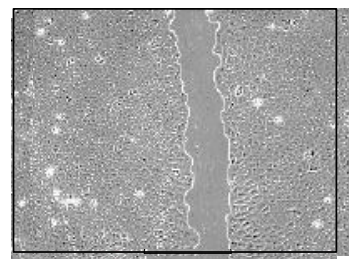

KO Clone 1
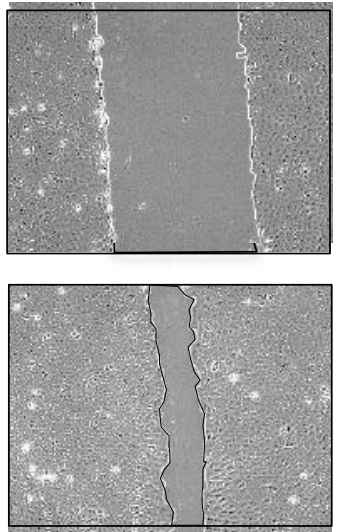

KO Clone 3
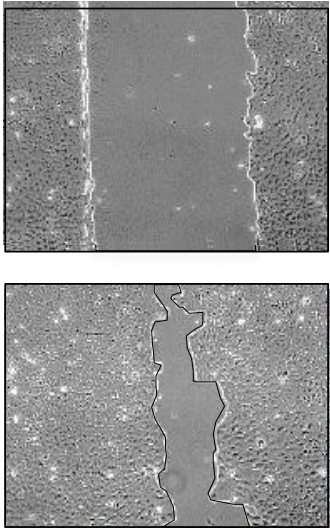

B

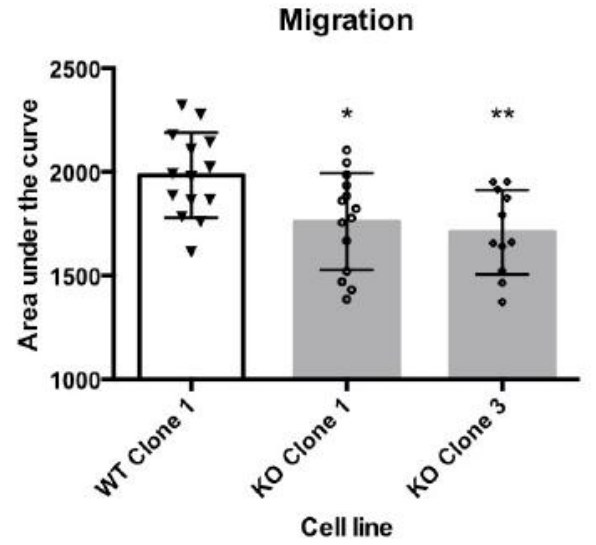

C

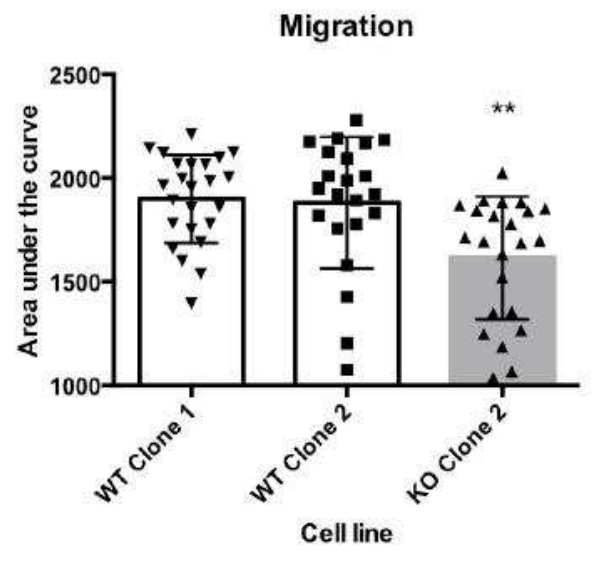

D

Proliferation

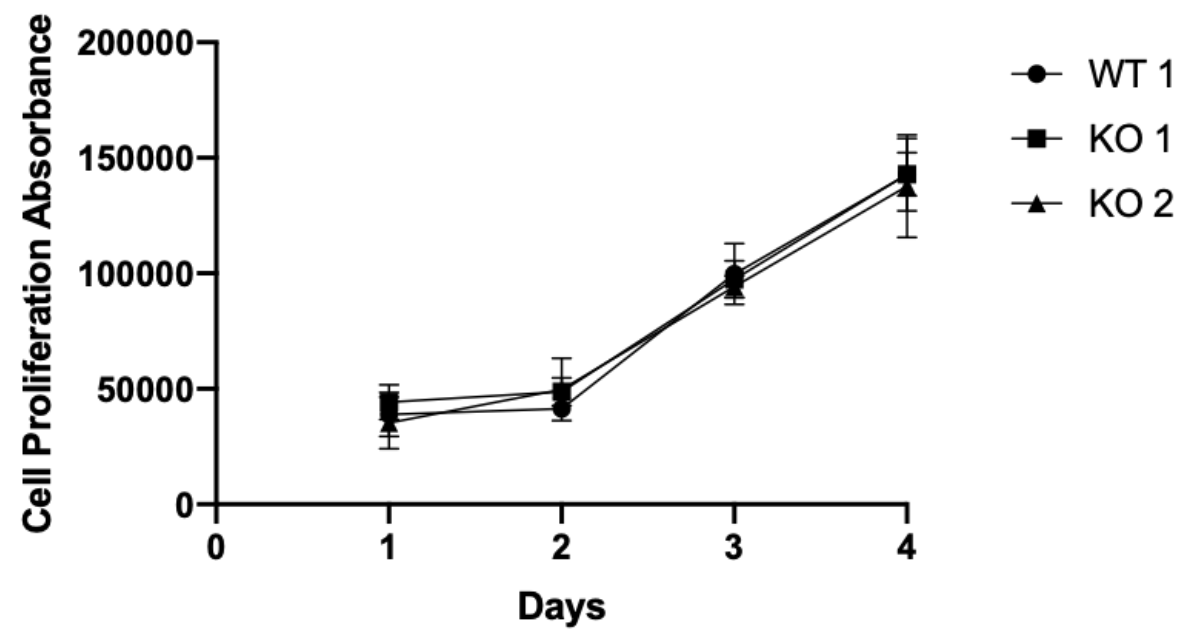


Figure 4

A

$\begin{array}{lllll}-1 & N & -1 & \mathfrak{n} & m \\ \xi & ⺊ & 0 & \hat{0} & \hat{0}\end{array}$

CIAP1

XIAP

Actin

B

$\begin{array}{lllllll}0.5 & 0.5 & 6 & 6 & 24 & 24 & \text { Time (h) }\end{array}$

$-\quad+\quad-\quad+\quad+\quad$ LCL 161

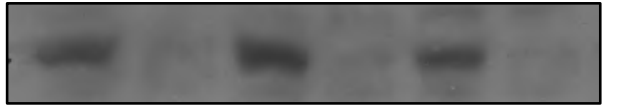

CIAP1

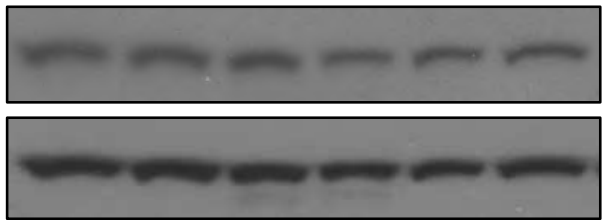

XIAP

Actin

C

Migration

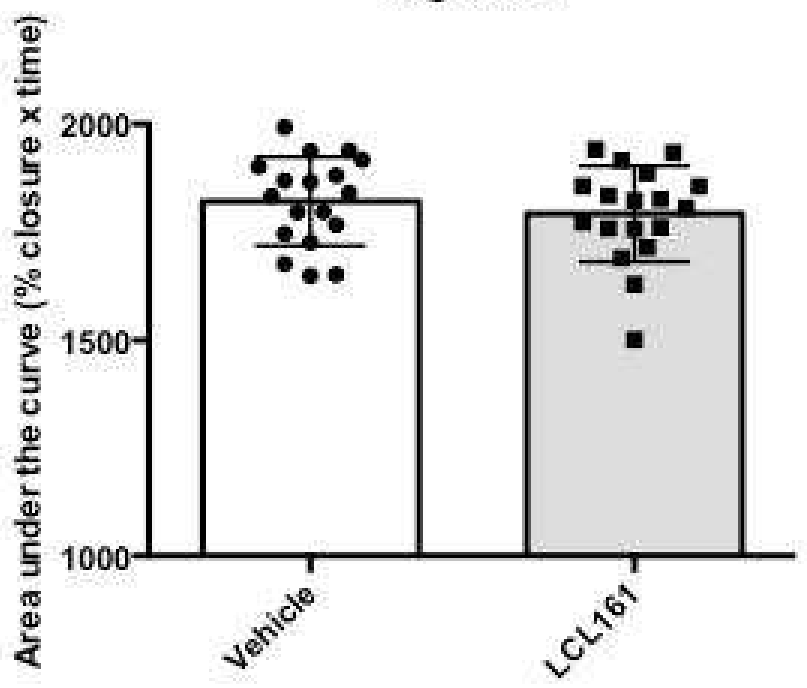


Figure 5

A

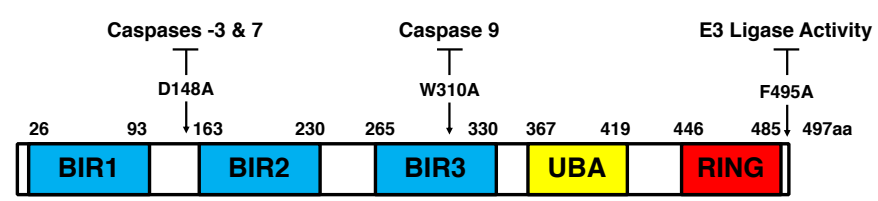

C

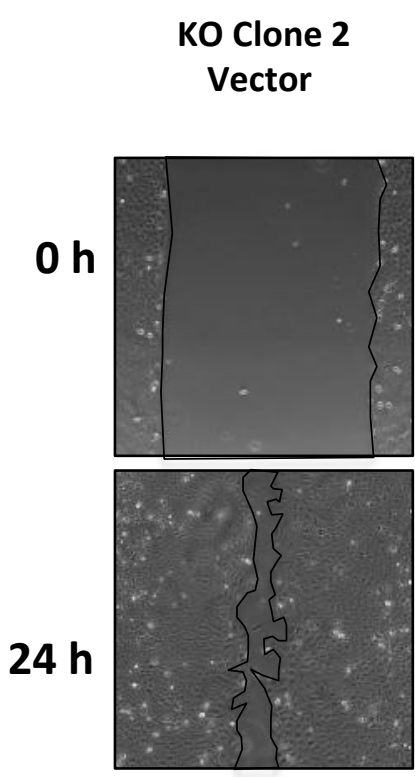

KO Clone 2 XIAP
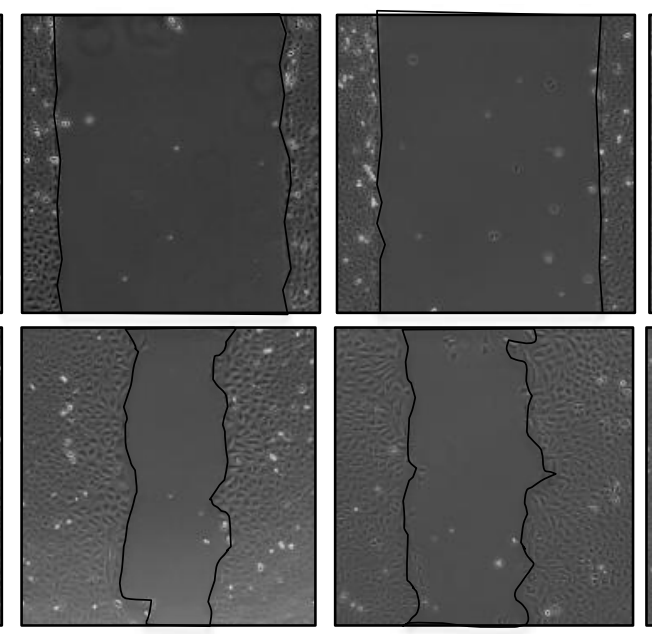
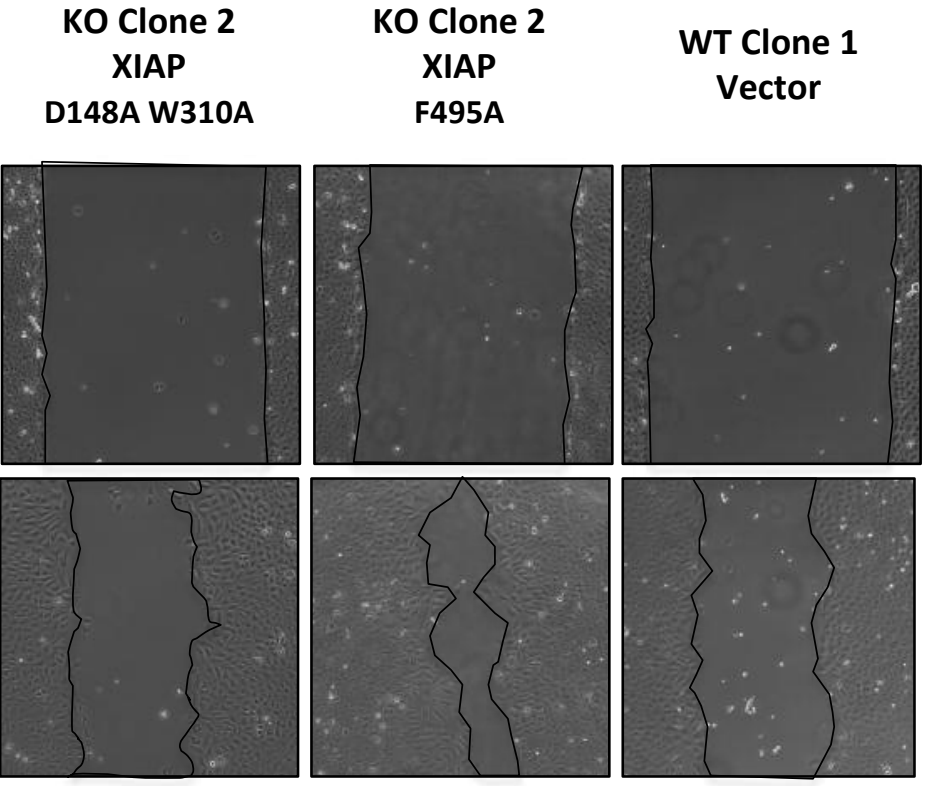

Actin

\section{Vector \\ XIAP WT \\ XIAP D148A/ W310A \\ XIAP F495A}

XIAP (BD)

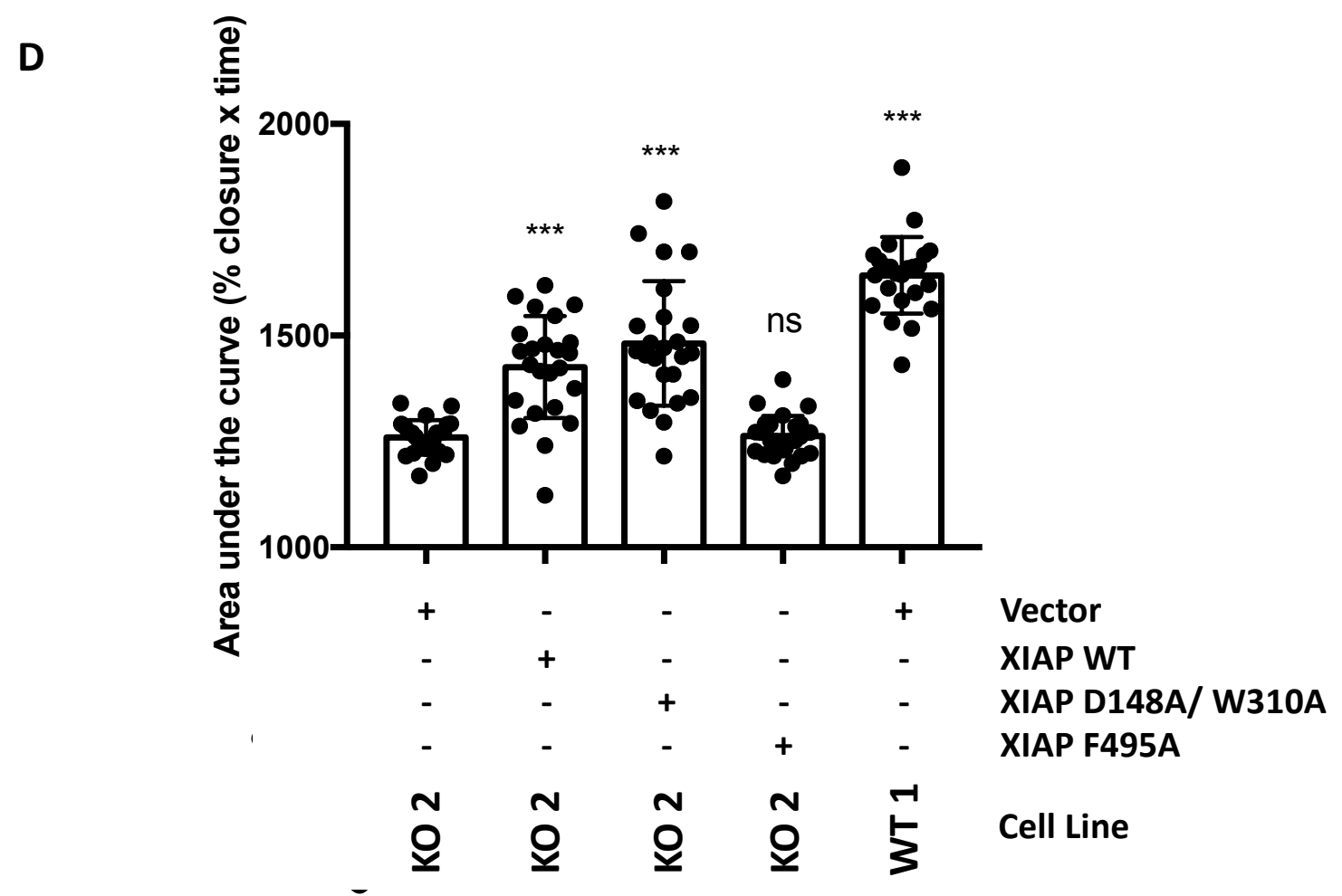


Figure 6

A

$\begin{array}{lllll}-1 & N & -1 & N & m \\ 3 & 5 & \underline{0} & \underline{0} & \underline{0}\end{array}$
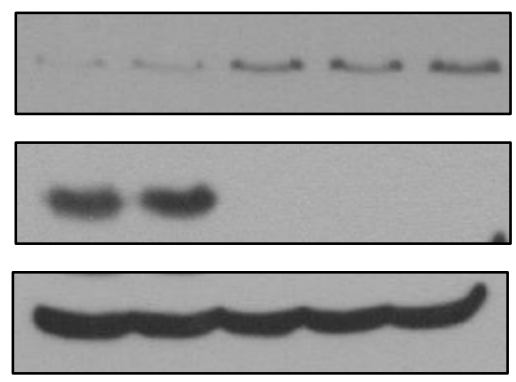

c $\quad \begin{array}{llll}\vec{g} & \tilde{g} & \vec{q} & \tilde{9}\end{array}$
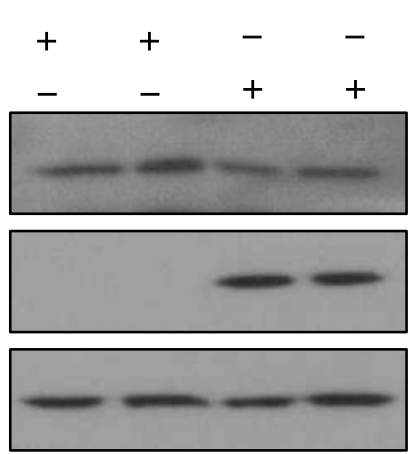

B

$\begin{array}{lllll}\vec{\xi} & \tilde{\xi} & \overrightarrow{9} & \tilde{9} & \stackrel{m}{9}\end{array}$

C-RAF

XIAP

Actin

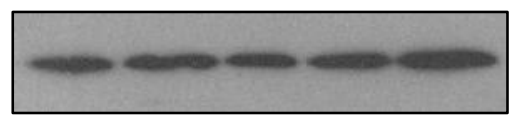

Cdc42

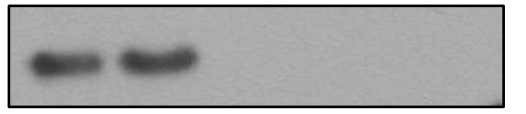

XIAP

Actin

Actin

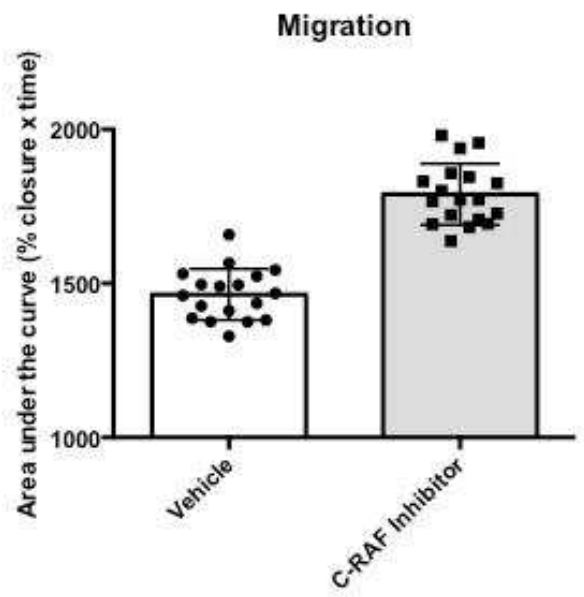




\section{Supplementary Figures}

Supplementary Figure 1. A. U2OS clonal cell lines were exposed to etoposide for 48 hours and viability was determined by PrestoBlue assay. The fluorescence intensity was measure and each data set were normalised to the reading from untreated cells. The normalised mean fluorescence intensity (MFI) at increasing concentrations of etoposide $(\log 2(\mathrm{mM}))$ for each clonal cell line is shown. Only data sets with a value of R2>0.85 were used and each curve represents the individual experimental repeats used to calculate the averages used in Figure 2A. B. As in (A), but using Doxorubicin as the cytotoxic agent.

Supplementary Figure 2. XIAP-null cells have increased migratory capacity. A Representative images of a wound healing experiment for wild type and XIAP null clones at 0 and 24 hours. The migration front is highlighted for clarity. B Representative images of the migration front for wild type and XIAP null clones at 0 and 24 hours. $C$ The length of the migration front was measured at 0 and $24 \mathrm{hr}$ using Image $\mathrm{J}$ software. The migration front length was plotted and analysed by unpaired students t-test.

\section{Supplementary Figure 3. XIAP-null cells have increased migratory capacity} A DNA sequence of the first exon of the XIAP gene with the TALEN binding sites indicated and allignments of DNA sequencing analysis of TALEN modified clonal U2OS cell lines. B PCR analysis of the genomic DNA of TALEN modified U2OS cell line using primers spanning the sites of TALEN-mediated cleavage. C. Whole cell lysates were prepared from TALEN-modified U2OS clonal cell lines. Lysates were subjected to immunoblot analysis to assess XIAP expression levels using a specific XIAP antibody (BD). D Representative images of a wound healing experiment for wild type and XIAP null clones at 0 and 24 hours. The migration front is highlighted for clarity. E The area of the wound was imaged and measured at 0,8 , and 24 hours using ImageJ software. The data was normalised with the 
percentage wound area equalling $100 \%$ at 0 hours. The $\%$ wound area was plotted against time and the area under the curve ( $\%$ wound area $x$ time(hours)) was calculated and analysed by unpaired students t-test.

\section{Supplementary Figure 4. XIAP-depleted HeLa cells have increased migratory} capacity. A HeLa cells transfected with the indicated siRNAs, whole-cell lysates (WCLs) prepared from these cells were subjected to immunoblot analysis to assess expression levels of the indicated proteins. B Representative images of a wound healing experiment for control and XIAP-depleted cells at 0 and 24 hours. The migration front is highlighted for clarity. The area of the wound was imaged and measured at 0 and 24 hours using ImageJ software. C The data was normalised with the percentage wound area equalling $100 \%$ at 0 hours. The $\%$ wound area was plotted against time and the area under the curve (\% wound area $x$ time(hours)) was calculated and analysed by unpaired students t-test. 24 scratches were measured (8 technical replicates from 3 independent experiments).

Supplementary Figure 5 XIAP controls levels of C-RAF to influence cell migration. The relative abundance of proteins A clAP1 B C-RAF C Cdc42 as compared to b-actin were quantified from 3 independent experiments in XIAP WT and $\mathrm{KO}$ cells using Image J software. Abundance was normalised to the mean of the 2 WT cell lines. Statistical analysis performed using a one-way ANOVA with Dunnett's multiple comparisons test and compared to the XIAP WT Clone 1. D. Relative abundance of C-RAF as compared to b-actin was quantified from 3 independent experimetns in XIAP KO cells transfected with empty vector or HAXIAP. Abundance was normalised to the mean of the XIAP KO cell lines transfected with empty vector. Statistical analysis performed using a one-way ANOVA with Dunnett's multiple comparisons test and compared to the XIAP KO Clone 1 transfected with empty vector. 
and was generated by quantifying blots from three independent experiments using Image $J$ and normalizing the intensity of the bands to the untreated lane 
A

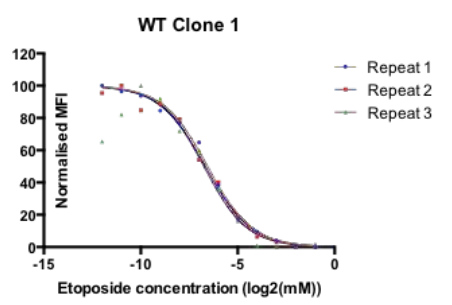

B

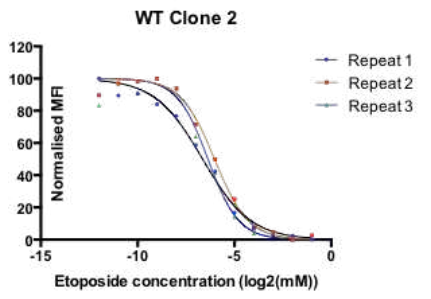

C

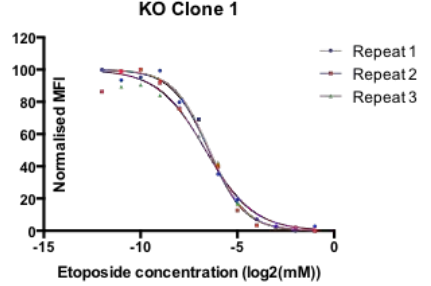

D

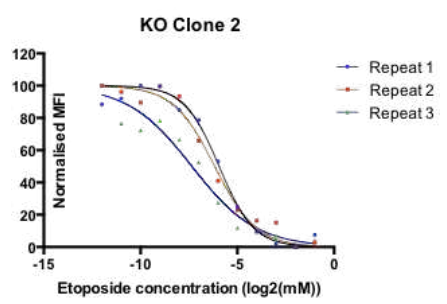

E

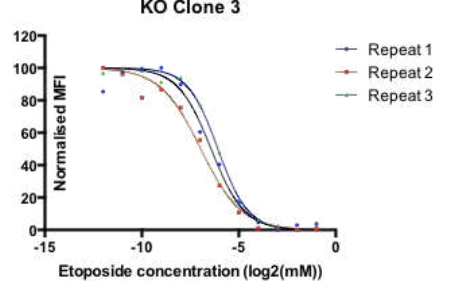

F

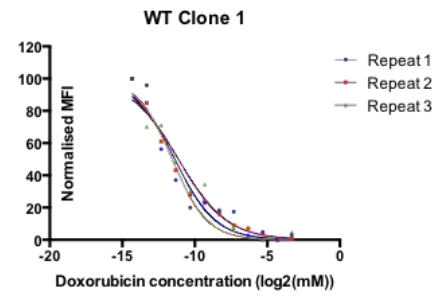

G

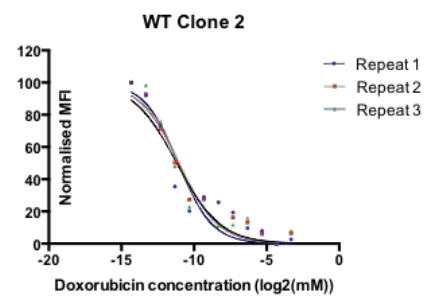

H

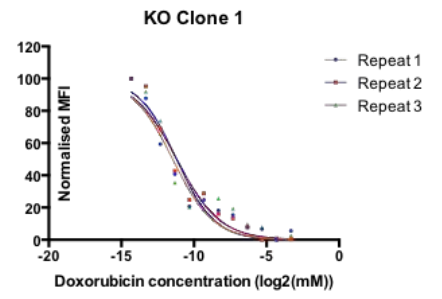

I

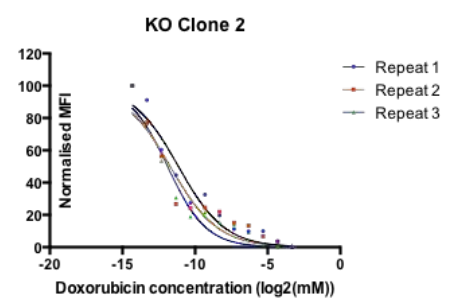

J

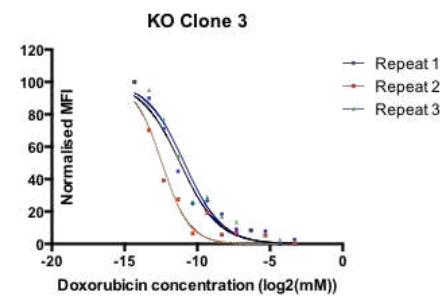


A

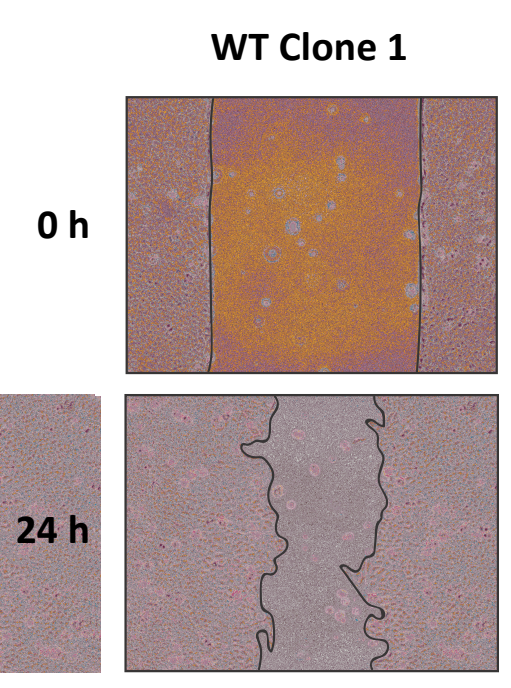

WT Clone 2

KO Clone 2

Sup Fig 2
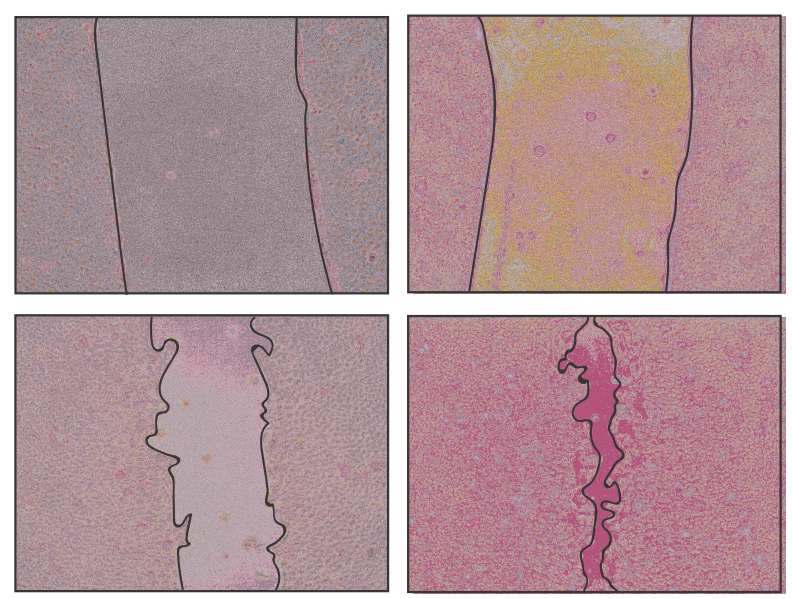

B

WT Clone 1
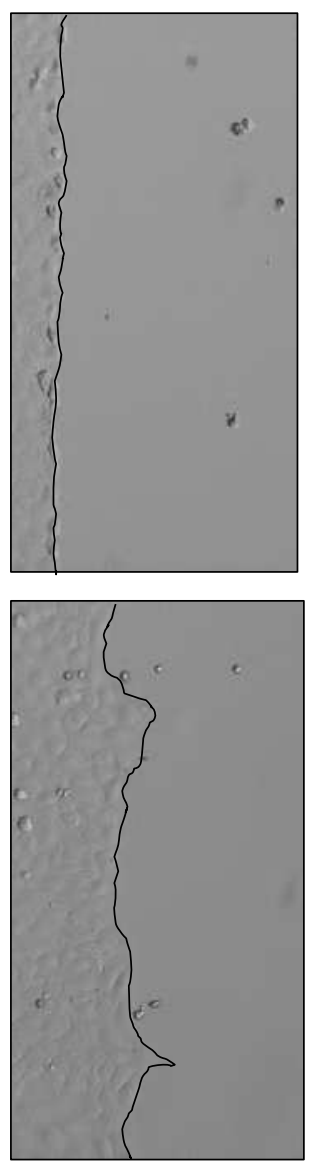

KO Clone 1
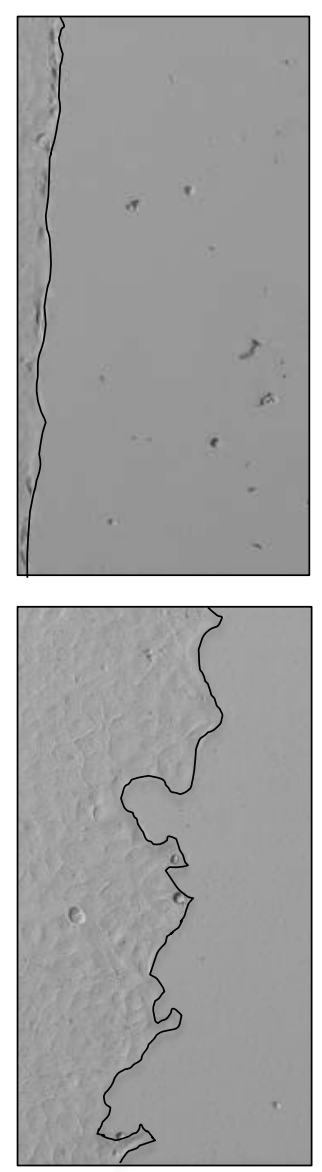

C

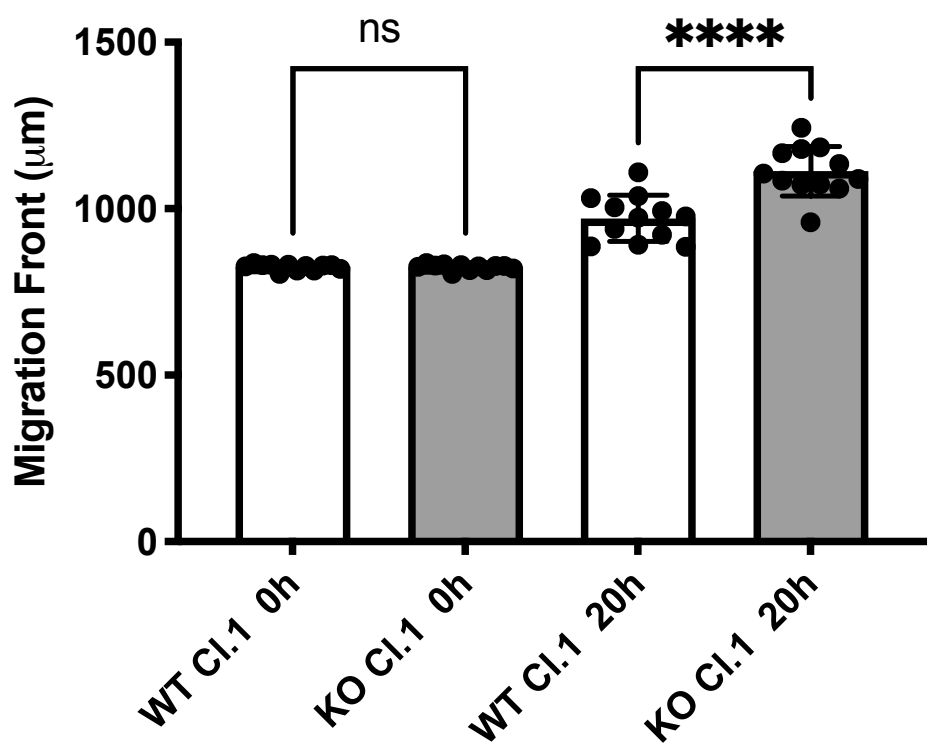

$20 \mathrm{~h}$ 
A

\section{TALEN Left}

TALEN Right

XIAP 100-ctaattttccaagtggtagtcctgtttcagcatcaacactggcacgagcagggtttctttatactggtgaaggagatacc-170

TALEN WT 1 100-ctaattttccaagtggtagtcctgtttcagcatcaacactggcacgagcagggtttcttatactggtgaaggagatacc-179

TALEN WT 2 100-ctaattttccaagtggtagtcctgtttcagcatcaacactggcacgagcagggtttctttatactggtgaaggagatacc-179

TALEN KO 1 100-ctaattttccaagtggtagtcctgtttcagcatcaacactggcacga----------tttatactggtgaaggagatacc-169

TALEN WT 3 100-ctaattttccaagtggtagtcctgtttcagcatcaacactggcacgagcagggtttctttatactggtgaaggagatacc-179

B

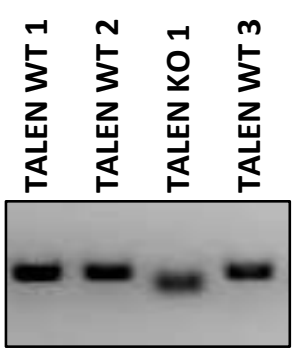

Diagnostic PCR
C

O h

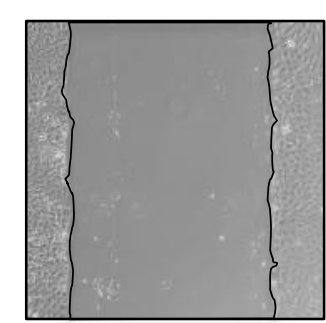

$24 \mathrm{~h}$

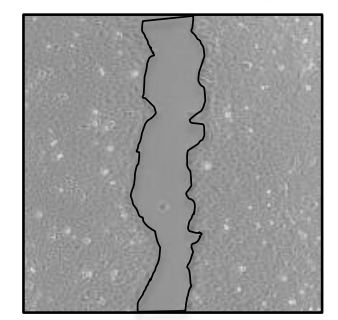

E

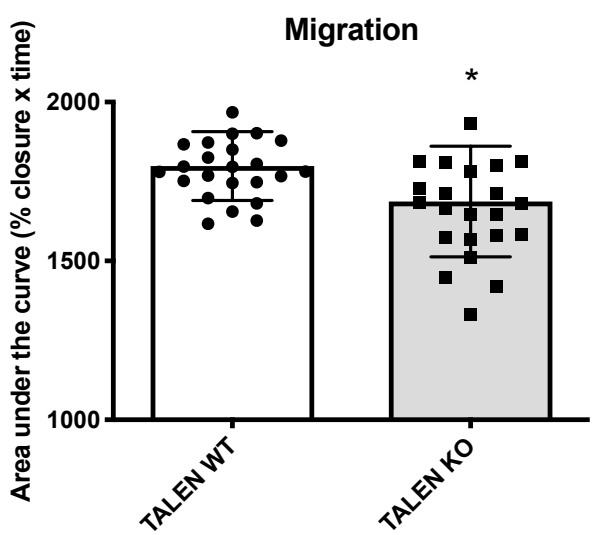

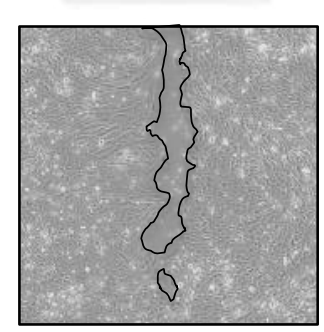

TALEN KO 1

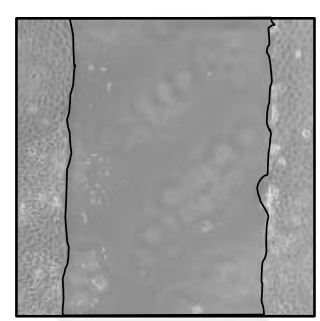

XIAP

Actin 


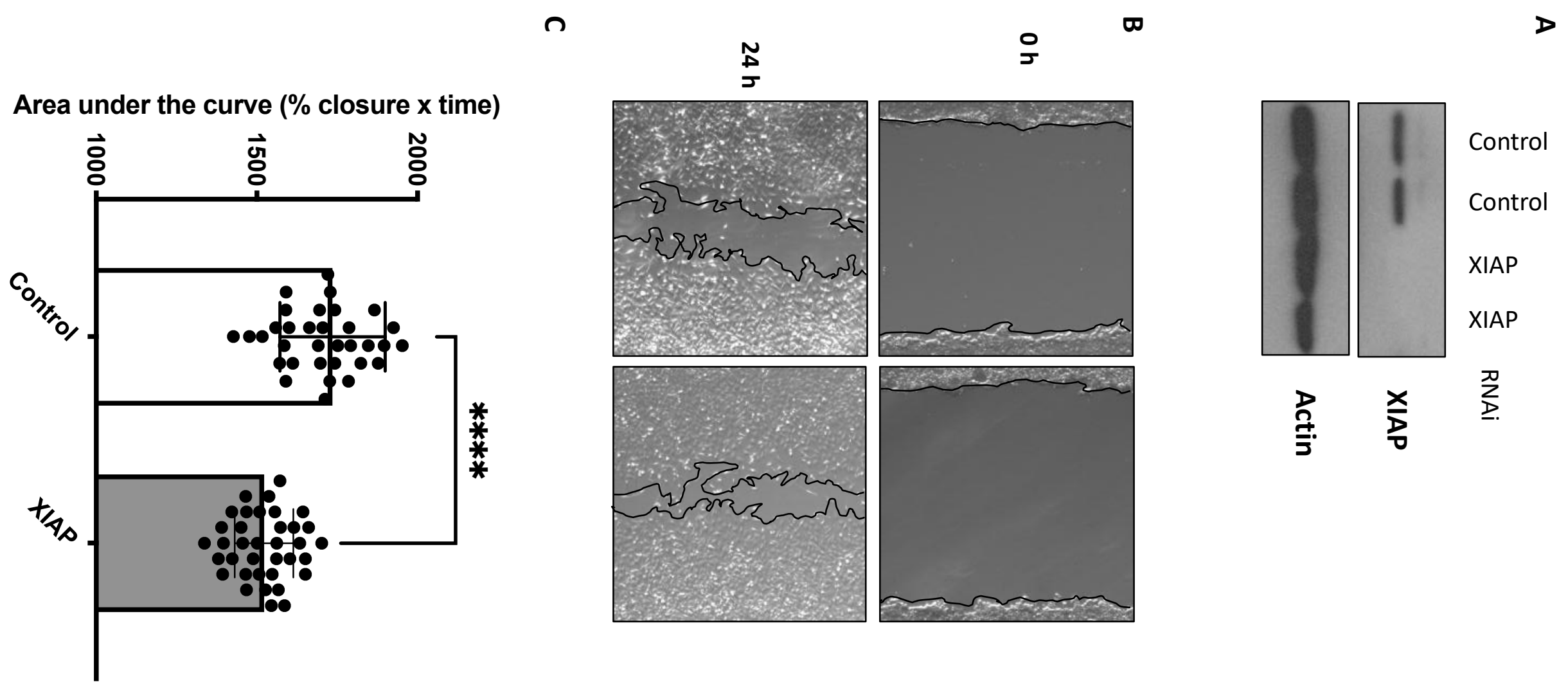



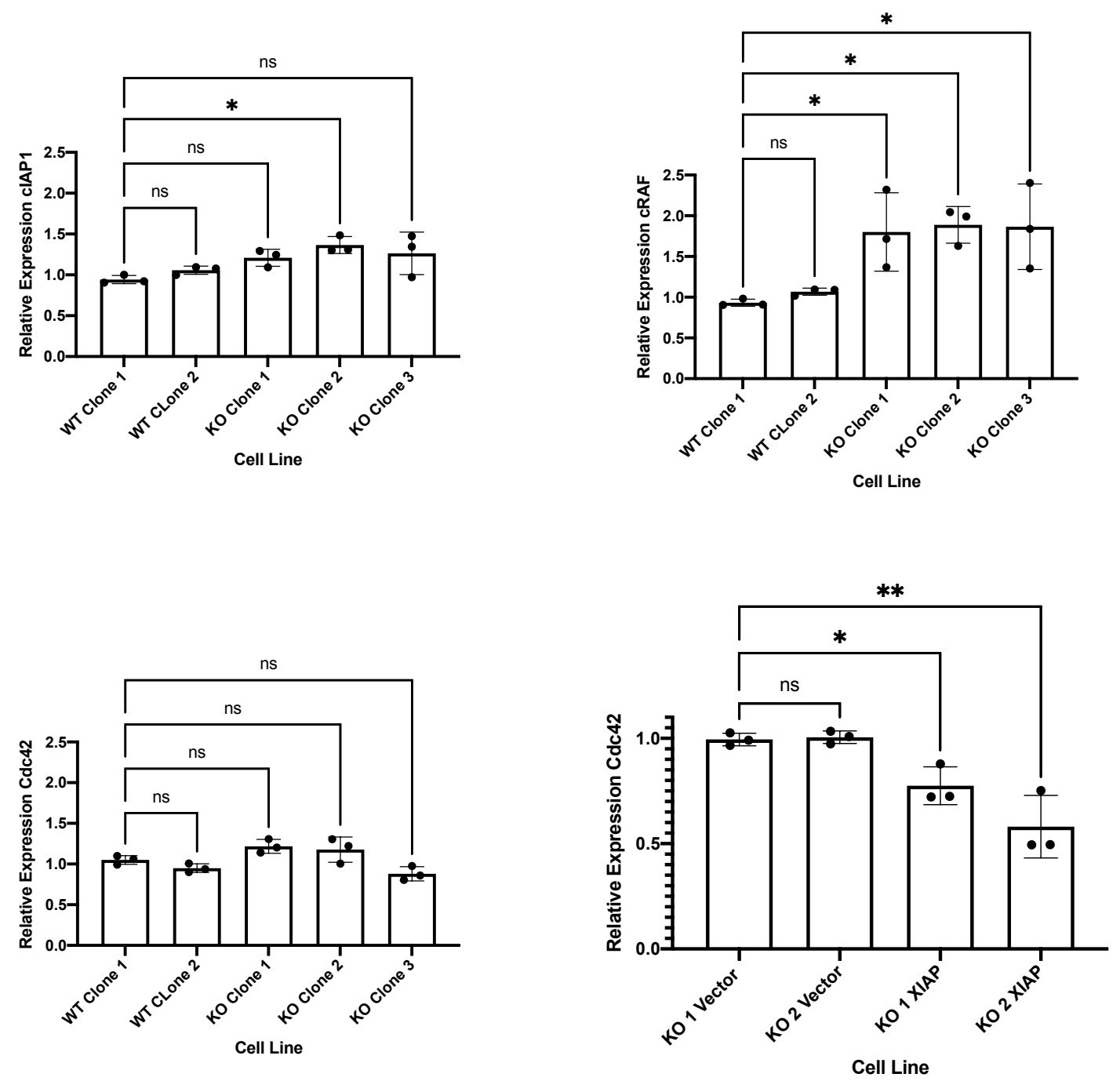
